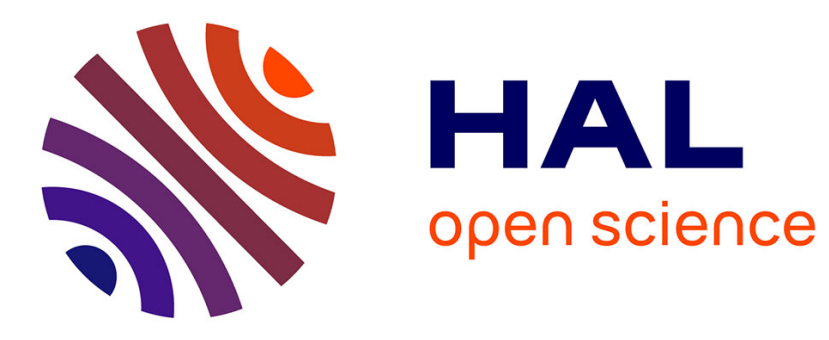

\title{
Numerical simulations of classical problems in two-dimensional (non) linear second gradient elasticity
}

Ugo Andreaus, Francesco Dell'Isola, Ivan Giorgio, Luca Placidi, Tomasz

Lekszycki, Nicola Luigi Rizzi

\section{- To cite this version:}

Ugo Andreaus, Francesco Dell'Isola, Ivan Giorgio, Luca Placidi, Tomasz Lekszycki, et al.. Numerical simulations of classical problems in two-dimensional (non) linear second gradient elasticity. International Journal of Engineering Science, 2016, 108, pp.34-50. hal-01378498

\section{HAL Id: hal-01378498 \\ https://hal.science/hal-01378498}

Submitted on 10 Oct 2016

HAL is a multi-disciplinary open access archive for the deposit and dissemination of scientific research documents, whether they are published or not. The documents may come from teaching and research institutions in France or abroad, or from public or private research centers.
L'archive ouverte pluridisciplinaire $\mathbf{H A L}$, est destinée au dépôt et à la diffusion de documents scientifiques de niveau recherche, publiés ou non, émanant des établissements d'enseignement et de recherche français ou étrangers, des laboratoires publics ou privés. 


\title{
Numerical simulations of classical problems in two-dimensional (non) linear second gradient elasticity
}

\author{
Ugo Andreaus a , Francesco dell'Isola ${ }^{a}$, Ivan Giorgio ${ }^{a}$, Luca Placidi ${ }^{b, *}$, \\ Tomasz Lekszycki ${ }^{c}$, Nicola Luigi Rizzi ${ }^{\mathrm{d}}$ \\ 'Universita di Roma La Sapienza, Department of structural and geotechnicol engineering. Italy \\ bintemationd Tetematic University Uninerano, Cso Vistorio Emonuete II, 3900186 Roma Itoby \\ 'Warsaw University of Tedholozy. Faculty of Engiseering Production Warsaw, Polend \\ ¿ Dipartimento di Architeture, Universitò degti studi Roma Tre, Rome, italy
}

\begin{abstract}
A B S T R A C T
A two-dimensional solid consisting of a linear elastic isotropic material is considered in this paper. The strain energy is expressed as a function of the strain and of the gradient of strain. The balance equations and the boundary conditions have been derived and numerically simulated for those classical problems for which an analytical solution is available in the literature. Numerical simulations have been developed with a commercial code and a perfect overlap between the results and the analytical solution has been found. The role of external edge double forces and external wedge forces has also been analyzed. We investigate a mesh-size independency of second gradient numerical solutions with respect to the classical first gradient one. The necessity of a second gradient modelling is finally shown. Thus, we analyze a non-linear anisotropic problem, for which experimental evidence of internal boundary layer is shown and we prove that this can be related to the second gradient modelling.
\end{abstract}

\section{Introduction}

The introduction of higher order gradients of the strain into the constitutive law for the internal energy leads to a partial differential equation of higher orders and the Galerkin method requires a higher regularity of the interpolation scheme, see e.g. Bilotta, Formica, and Turco (2010). The reason of introducing higher-order gradient theories is based on different points of view, see e.g. Aminpour and Rizzi (2015, 2016a,b); Aminpour, Rizzi, and Salerno (2014). The first example is referred to the case related to strongly localized deformation features (Altenbach, Eremeyev, and Morozov, 2010; Cuomo, Contrafatto, and Greco, 2014; Luca, 2015; Luca, 2016; Roveri, Carcaterra, \& Akay, 2009) and references therein. In such cases, it is reasonable to complement the displacement field with some additional kinematical descriptors (Gabriele, Rizzi, \& Varano, 2012, 2016. 2014; Piccardo, Ranzi, \& Luongo, 2014a,b: Pigmataro, Ruta, Rizzi, \& Varano, 2010; Rizzi \& Varano, 2011). which leads to the so-called micromorphic models, see also lonel-Dumitrel, Patrizio, Angela, Placidi, and Giuseppe (2015); Madeo, Neff, Ghiba, Placidi, and Rosi (2015a,b); Neff, Ghiba, Madeo, Placidi, and Rosi (2014). Another possibility is to consider higher order gradient theories, in which the deformation energy depends on second and/or higher gradients of the

\footnotetext{
- Corresponding author, Fax: +390669207620

E-mait address: lucaplacidipuninettunouniversity.net ( $L$ Placidi).
} 
displacement (dell'Isola, Madeo, \& Placidi, 2012; Ferretti, Madeo, dell'Isola, \& Boisse, 2014; Madeo, dell'Isola, \& Darve, 2013). This is done in the literature for both monophasic systems (see Auffray, dell'Isola, Eremeyev, Madeo, and Rosi, 2015; Rosi, Giorgio, and Eremeyev, 2013, in which continuous systems are investigated, and Alibert and Corte (2015); Seppecher, Alibert, and dell'Isola (2011) for cases of lattice/woven structures) and for bi-phasic (see e.g. Andreaus, Giorgio, \& Lekszycki, 2014; dell'Isola, Guarascio, \& Hutter, 2000; Misra, Parthasarathy, Singh, \& Spencer, 2015; Sciarra, dell'Isola, \& Coussy, 2007) or granular materials (Misra \& Singh, 2014; Yang \& Misra, 2012). The second example is referred to the fact that, unlike classical Cauchy continua, second and higher order continua can respond to concentrated forces and generalized contact actions (see e.g. in Carcaterra \& Roveri, 2012; de Oliveira Góes, de Castro, \& Martha, 2014). It is worth to be noted that it is also possible to conceive a framework in classical elasticity (see, e.g., Bulíček, Málek, Rajagopal, and Walton, 2015; Mindlin, 1936 and references therein) in which concentrated forces are possible. However, with a greater theoretical and numerical efforts. The third example is becoming increasingly important for practical and applicative reasons in the last years, as the novelties in manufacturing procedures (due to, e.g., 3D printing, self assembly etc.) are making possible the realization of a much wider class of new architectured materials (Del Vescovo \& Giorgio, 2014; Milton \& Seppecher, 2012). In these cases, the deficiencies of classical approaches when the material behaviour exhibits size-scale effects is investigated in Sansour and Skatulla (2009); Scerrato, Giorgio, and Rizzi (2016); Scerrato, Zhurba Eremeeva, Lekszycki, and Rizzi (2016), and in Neff, Jeong, and Ramézani (2009) a novel invariance requirement (micro-randomness) in addition to isotropy is formulated, which implies conformal invariance of the curvature. In general, new theories are put into place when existing theories prove to be inadequate to describe some observed phenomenon. Such new theories however have to lead to well posed problems in the sense that the governing equations and boundary conditions lead to solvable problems. The papers (Alibert \& Corte, 2015; Mareno \& Healey, 2006; Pideri \& Seppecher, 1997) already proved that the problems we study here is indeed well-posed.

A survey of variational principles, which form the basis for computational methods in both continuum mechanics and multi-rigid body dynamics is presented in Atluri and Cazzani (1995) and numerical investigation of structures of the type considered also requires special attention and the development of novel techniques (Assante \& Cesarano, 2014; Bilotta \& Turco, 2009; Cesarano, 2014; Cesarano \& Assante, 2014; Cesarano, Fornaro, \& Vazquez, 2016; Garusi, Tralli, \& Cazzani, 2004; Greco \& Cuomo, 2015) or the proper employment of the existing ones (see for instance Terravecchia, Panzeca, and Polizzotto, 2014, where Galerkin Boundary Element Method is used to address a class of strain gradient elastic materials). The objective of the contribution (Javili, Dell'Isola, \& Steinmann, 2013) is to formulate a geometrically nonlinear theory of higher-gradient elasticity accounting for boundary (surface and curve) energies. To reduce the computational costs and avoid the macroscopic grid sensitivity, an adaptive multiscale technique is developed for strain localization analysis of periodic lattice truss materials in Zhang, Wu, and Zheng (2012). In Zervos (2008) a general finite element discretization of micromorphic Mindlin' s elasticity is presented. The behavior of all elements is also examined at the limiting case of strain gradient elasticity. The numerical solution of second gradient elasticity equations with a displacement-based finite element method requires the use of C1-continuous elements, that motivates the implementation of the concept of isogeometric analysis in Fischer, Klassen, Mergheim, Steinmann, and Müller (2011). In Papanicolopulos, Zervos, and Vardoulakis (2009) a new C1 hexahedral element which is the first three-dimensional $\mathrm{C} 1$ element ever constructed and give excellent rates of convergence in a benchmark (without edge forces) boundary value problem of gradient elasticity. In Papanicolopulos and Zervos (2012) a methodology by which C1 elements, such as the TUBA 3 element proposed by Argyris, Fried, and Scharpf (1968), can be constructed is presented. This kind of elements are largely present in the literature of strain gradient elasticity (Akarapu \& Zbib, 2006; Dasgupta \& Sengupta, 1990; Fischer, Mergheim, \& Steinmann, 2010; Zervos, Papanastasiou, \& Cook, 1998; Zervos, Papanastasiou, \& Vardoulakis, 2001; Zervos, Papanicolopulos, \& Vardoulakis, 2009).

From a general point of view a comparison between analytical and numerical solution is needed to check the quality of the used code. In other words, it means that the code has good performances and there is a degree of reliability to be assigned to it. In this paper a two-dimensional solid consisting of a linear elastic isotropic material is considered. The strain energy is expressed as a function of the strain and of the gradient of strain. The aim of the paper is to present the possibility to numerically simulate general strain gradient elasticity by the use of a commercial code that includes the Argyris shape functions. This is done with the use of benchmark boundary value problem for which an analytical solution exist. We remark that in such a 2-dimensional benchmark boundary value problem wedge forces are present. We remark a perfect overlap between the numerical results and the analytical solution of the benchmark classical boundary value problem. The role of external edge double forces and external wedge forces has also been analyzed. We also investigate a mesh-size independency of second gradient numerical solutions with respect to the classical first gradient one.Finally, we show an experimental evidence of the necessity of second gradient modeling. We show the experimental results (dell'Isola, Giorgio, Pawlikowski, \& Rizzi, 2016) of a bias test on a pantographic structure. In particular, we show that the boundary layer experimentally observed can be numerically achieved by a non-null second gradient constitutive coefficient and the largeness of such a boundary layer can be used to identify such a second gradient parameter. For a better representation of the state of the art of material identification of second gradient coefficients the reader is invited to see Auffray, Bouchet, and Brechet (2009); Luca, Andreaus, Corte, and Lekszycki (2015); Placidi, Andreaus, and Giorgio (2016). 


\section{Formulation of the problem}

\subsection{The general case}

The coordinates $X$, in the reference configuration, are those of the 2-dimensional body $\mathcal{B}$. The internal energy density functional $U(G, \nabla G)$ depends not only on the strain $G=\left(F^{T} F-I\right) / 2$ but also on its gradient $\nabla G$, where $F=\nabla \chi, \chi$ is the placement function. In Mindlin (1964) a general form of the density of the strain energy functional of a linear isotropic second gradient elastic material is given, for the sake of simplicity, in indicial notation,

$$
U(G, \nabla G)=\frac{\lambda}{2} G_{i i} G_{j j}+\mu G_{i j} G_{i j}+4 \alpha_{1} G_{a a, b} G_{b c, c}+\alpha_{2} G_{a a, b} G_{c c, b}+4 \alpha_{3} G_{a b, a} G_{c b, c}+2 \alpha_{4} G_{a b, c} G_{a b, c}+4 \alpha_{5} G_{a b, c} G_{a c, b},
$$

where subscript $j$ after comma indicates derivative with respect to $X_{j}$ and a general rule for index notation is the following: the subscript-indices of a symbol denoting a vector or a tensor quantity denote the components of that quantity. In the 2-dimensional case we have

$$
\begin{aligned}
U(G, \nabla G)= & \tilde{U}(u)=(\lambda+2 \mu)\left(u_{1,1}^{2}+u_{2,2}^{2}\right)+\mu\left(u_{1,2}^{2}+u_{2,1}^{2}\right)+2 \lambda u_{1,1} u_{2,2}+2 \mu u_{1,2} u_{2,1} \\
& +\frac{1}{2} A\left(u_{1,22}^{2}+u_{2,11}^{2}\right)+\frac{1}{2} B\left(u_{1,11}^{2}+u_{2,22}^{2}\right)+C\left(u_{1,12}^{2}+u_{2,12}^{2}\right)+2 D\left(u_{1,11} u_{2,12}+u_{2,22} u_{1,12}\right) \\
& +\frac{1}{2}(A+B-2 C)\left(u_{1,11} u_{1,22}+u_{2,11} u_{2,22}\right)+(B-A-2 D)\left(u_{1,12} u_{2,11}+u_{1,22} u_{2,12}\right),
\end{aligned}
$$

where

$$
\begin{aligned}
& A=2 \alpha_{3}+2 \alpha_{4}+2 \alpha_{5}, \quad B=8 \alpha_{1}+2 \alpha_{2}+8 \alpha_{3}+4 \alpha_{4}+8 \alpha_{5} . \\
& C=2 \alpha_{1}+\alpha_{2}+\alpha_{3}+3 \alpha_{4}+5 \alpha_{5}, \quad D=3 \alpha_{1}+\alpha_{2}+2 \alpha_{3},
\end{aligned}
$$

where $u$ is the displacement field, $\lambda$ and $\mu$ are the Lamè coefficients and $\alpha_{i}$ with $i=1,2,3,4,5$ are the 5 second gradient constitutive parameters. Note that here we use the Lamè coefficients $\lambda$ and $\mu$ to describe first-gradient, isotropic linear elasticity, but other choices could be made, e.g., the pair comprised of bulk modulus $\kappa$ and shear modulus $\mu$ (Federico, Grillo, \& Herzog, 2004; Federico, Grillo, \& Imatani, 2015; Federico, Grillo, \& Wittum, 2009; Hill, 1965; Walpole, 1981, 1984), which is particularly convenient, e.g., when treating quasi-incompressibility.

In Mindlin (1964), in order to have the positive definiteness of $U$, the following restrictions on the 7 constitutive parameters must be satisfied,

$$
\begin{aligned}
& \mu>0, \quad 3 \lambda+2 \mu>0, \quad-4 \alpha_{1}+2 \alpha_{2}+2 \alpha_{3}+6 \alpha_{4}-6 \alpha_{5}>0, \quad \alpha_{4}>\alpha_{5}, \quad \alpha_{4}+2 \alpha_{5}>0 \\
& 4 \alpha_{1}+\alpha_{2}+4 \alpha_{3}+2 \alpha_{4}+4 \alpha_{5}>0, \quad \alpha_{1}+\alpha_{2}<\alpha_{3}, \quad 4 \alpha_{1}-2 \alpha_{2}-2 \alpha_{3}-3 \alpha_{4}+3 \alpha_{5}>0 .
\end{aligned}
$$

In the 2-dimensional case the positive definiteness of $U$ is implied again by the classical 2-dimensional restrictions

$$
\mu>0, \quad \lambda+\mu>0,
$$

and by the positive definiteness of the following matrix

$$
\left(\begin{array}{cccccc}
A & 0 & \frac{1}{2}(A+B-2 C) & 0 & 0 & B-A-2 D \\
0 & A & 0 & \frac{1}{2}(A+B-2 C) & B-A-2 D & 0 \\
\frac{1}{2}(A+B-2 C) & 0 & B & 0 & 0 & 2 D \\
0 & \frac{1}{2}(A+B-2 C) & 0 & B & 2 D & 0 \\
0 & B-A-2 D & 0 & 2 D & 2 C & 0 \\
B-A-2 D & 0 & 2 D & 0 & 0 & 2 C
\end{array}\right) .
$$

Keeping this in mind, a classical variational procedure gives the following system of partial differential equations $\forall X_{i} \in \mathcal{B}$,

$$
\begin{aligned}
& u_{1,11}(\lambda+2 \mu)+u_{1,22} \mu+u_{2,12}(\lambda+\mu) \\
& \quad=u_{1,1111} B+u_{1,2222} A+u_{1,1122}(A+B)+\left(u_{2,1222}+u_{2,1112}\right)(B-A)-b_{1}^{\text {ext }} \\
& u_{2,22}(\lambda+2 \mu)+u_{2,11} \mu+u_{1,12}(\lambda+\mu) \\
& \quad=u_{2,2222} B+u_{2,1111} A+u_{2,1122}(A+B)+\left(u_{1,1222}+u_{1,1112}\right)(B-A)-b_{2}^{\text {ext }},
\end{aligned}
$$

and boundary conditions given $\forall X_{i} \in \partial \mathcal{B}$ from the following duality conditions

$$
\delta u_{\alpha}\left(t_{\alpha}-t_{\alpha}^{e x t}\right)=0, \delta u_{\alpha, j} n_{j}\left(\tau_{\alpha}-\tau_{\alpha}^{e x t}\right)=0, \int_{[\partial \partial \mathcal{B}]} \delta u_{\alpha} f_{\alpha}=\int_{[\partial \partial \mathcal{B}]} \delta u_{\alpha} f_{\alpha}^{e x t},
$$

where $b_{\alpha}^{\text {ext }}, t_{\alpha}^{\text {ext }}, \tau_{\alpha}^{\text {ext }}$ and $f_{\alpha}^{\text {ext }}$ are the external actions: $b_{\alpha}^{\text {ext }}$ is the external force per unit area and is applied on the whole 2-dimensional domain $\mathcal{B} ; t_{\alpha}^{\text {ext }}$ and $\tau_{\alpha}^{\text {ext }}$ are the external force and double force (respectively) and are applied on (a part 
of) the one-dimensional boundary $\partial \mathcal{B}$ of the domain $\mathcal{B}$; and $f_{\alpha}^{\text {ext }}$ is the external concentrated force applied on the set of points belonging to the boundary of the boundary $[\partial \partial \mathcal{B}]$, so that the last integral can be also represented as the sum of the external works made by the concentrated forces acting on each vertex of the domain. In other words, if we define the boundary $\partial \mathcal{B}$ as the union of $m$ regular parts $\Sigma_{c}$ with $c=1, \ldots, m$ and $[\partial \partial \mathcal{B}]$ as the union of the corresponding $m$ vertex-points $\mathcal{V}_{c}$ with $c=1, \ldots, m$,

$$
\partial \mathcal{B}=\bigcup_{c=1}^{m} \Sigma_{c}, \quad[\partial \partial \mathcal{B}]=\bigcup_{c=1}^{m} \mathcal{V}_{c},
$$

then the line and vertex-integrals of a generic field $g\left(X_{i}\right)$ are represented as follows,

$$
\oint_{\partial \mathcal{B}} g\left(X_{i}\right)=\sum_{c=1}^{m} \int_{\Sigma_{c}} g\left(X_{i}\right), \quad \int_{[\partial \partial \mathcal{B}]} g\left(X_{i}\right)=\sum_{c=1}^{m} g\left(X_{i}^{c}\right)
$$

where $X_{i}^{c}$ is the coordinate of the vertex $\mathcal{V}_{c}$. Moreover, the so called contact force $t_{\alpha}$, contact double force $\tau_{\alpha}$ and contact wedge force $f_{\alpha}$ are defined,

$$
\begin{aligned}
t_{\alpha} & =\left(S_{\alpha j}-T_{\alpha j h, h}\right) n_{j}-P_{k a}\left(T_{\alpha h j} P_{a h} n_{j}\right)_{, k} \\
\tau_{\alpha} & =T_{\alpha j k} n_{j} n_{k} \\
f_{\alpha} & =T_{\alpha h k} V_{h k}
\end{aligned}
$$

where $n_{i}$ is the normal to the boundary $\partial \mathcal{B}, P_{i j}$ is its tangential projector operator $\left(P_{i j}=\delta_{i j}-n_{i} n_{j}\right), V$ is the vertex operator

$$
V_{h j}=v_{h}^{l} n_{j}^{l}+v_{h}^{r} n_{j}^{r}
$$

where superscripts $l$ and $r$ refers (roughly speaking, left and right), respectively, to one and to the other sides that define a certain vertex-point $\mathcal{V}_{c} ; v$ is the external tangent unit vector. Stress and hyper stresses are defined,

$$
S_{i j}=\frac{\partial U}{\partial G_{i j}}, \quad T_{i j h}=\frac{\partial U}{\partial G_{i j, h}} .
$$

\subsection{Rectangles}

\subsubsection{The general case of straight lines}

In the case of boundaries $\partial \mathcal{B}$ composed of straight-lines, the contact force in (10), the contact double force in (11) and the contact wedge force (12) are

$$
t_{\alpha}=S_{\alpha j} n_{j}-\left(T_{\alpha j h, h}+T_{\alpha h j, h}\right) n_{j}+T_{\alpha h j, k} n_{h} n_{k} n_{j}, \tau_{\alpha}=T_{\alpha j k} n_{j} n_{k}, f_{\alpha}=T_{\alpha i j} V_{i j},
$$

that, in terms of the displacement fields, yield,

$$
\begin{aligned}
& t_{\alpha}=\lambda u_{a, a} n_{\alpha}+\mu u_{\alpha, j} n_{j}+\mu u_{j, \alpha} n_{j}-u_{a, a b b} n_{\alpha}\left(6 \alpha_{1}+2 \alpha_{2}+4 \alpha_{3}\right) \\
& -u_{a, a \alpha k} n_{k}\left(6 \alpha_{1}+2 \alpha_{2}+4 \alpha_{3}+2 \alpha_{4}+8 \alpha_{5}\right)-u_{\alpha, a a k} n_{k}\left(2 \alpha_{3}+4 \alpha_{4}+6 \alpha_{5}\right) \\
& -u_{k, \alpha a a} n_{k}\left(2 \alpha_{1}+2 \alpha_{3}+2 \alpha_{4}+6 \alpha_{5}\right)+u_{a, a j k} n_{\alpha} n_{j} n_{k}\left(4 \alpha_{1}+2 \alpha_{2}+2 \alpha_{3}\right) \\
& +u_{j, a a k} n_{\alpha} n_{j} n_{k}\left(2 \alpha_{1}+2 \alpha_{3}\right)+u_{\alpha, a b c} n_{a} n_{b} n_{c}\left(2 \alpha_{4}+2 \alpha_{5}\right)+u_{a, \alpha b c} n_{a} n_{b} n_{c}\left(2 \alpha_{4}+6 \alpha_{5}\right), \\
& \tau_{\alpha}=u_{a, a b} n_{\alpha} n_{b}\left(4 \alpha_{1}+2 \alpha_{2}+2 \alpha_{3}\right)+u_{a, b b} n_{\alpha} n_{a}\left(2 \alpha_{1}+2 \alpha_{3}\right) \\
& +\left(2 \alpha_{1}+2 \alpha_{3}\right) u_{a, a \alpha}+u_{\alpha, a b} n_{a} n_{b}\left(2 \alpha_{4}+2 \alpha_{5}\right)+2 \alpha_{3} u_{\alpha, a a}+u_{a, \alpha b} n_{a} n_{b}\left(2 \alpha_{4}+6 \alpha_{5}\right) .
\end{aligned}
$$

We remark that the formulation expressed in (15) and (16) can also be used in the 3-dimensional case. This is the reason why (15) and (16) are expressed in terms of the 53 -dimensional constitutive coefficients $\alpha_{i}$ with $i=1,2,3,4,5$ and not in terms of the 42 -dimensional constitutive coefficients $A, B, C$ and $D$.

In Fig. 1 we represent the scheme of a rectangle with side-names $Q R, S$ and $T$ and vertex-names $V_{1}, V_{2}, V_{3}$ and $V_{4}$.

\subsubsection{Characterization of sides} have

The characterization of side $S$ is done by setting $n_{i}=\delta_{i 1}$. Thus, from (15) with $\alpha=1,2$, and from (16) with $\alpha=1,2$, we

$$
\begin{aligned}
& t_{1}=t_{1}^{S}=u_{1,1}(\lambda+2 \mu)+u_{2,2} \lambda-B u_{1,111}-2 D u_{2,222}-\frac{1}{2}(A+B+2 C) u_{1,122}-(B-A) u_{2,211}, \\
& t_{2}=t_{2}^{S}=\mu\left(u_{1,2}+u_{2,1}\right)-(B-A) u_{1,112}-(B-A-2 D) u_{1,222}-A u_{2,111}-\frac{1}{2}(A+B+2 C) u_{2,122},
\end{aligned}
$$




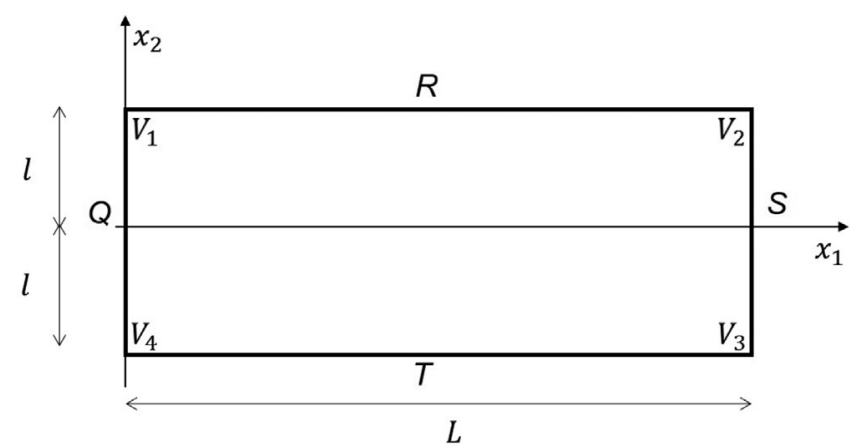

Fig. 1. Picture of the 2-dimensional body $\mathcal{B}$.

$$
\begin{aligned}
& \tau_{1}=\tau_{1}^{S}=B u_{1,11}+\frac{1}{2}(A+B-2 C) u_{1,22}+2 D u_{2,12}, \\
& \tau_{2}=\tau_{2}^{S}=(B-A-2 D) u_{1,12}+A u_{2,11}+\frac{1}{2}(A+B-2 C) u_{2,22} .
\end{aligned}
$$

The characterization of side $Q$ is done by setting $n_{i}=-\delta_{i 1}$. Thus, from (15) with $\alpha=1,2$, and from (16) with $\alpha=1,2$, we have

$$
\begin{aligned}
& t_{1}=t_{1}^{Q}=-u_{1,1}(\lambda+2 \mu)-u_{2,2} \lambda+B u_{1,111}+2 D u_{2,222}+\frac{1}{2}(A+B+2 C) u_{1,122}+(B-A) u_{2,211} \\
& t_{2}=t_{2}^{Q}=-\mu\left(u_{1,2}+u_{2,1}\right)+(B-A) u_{1,112}+(B-A-2 D) u_{1,222}+A u_{2,111}+\frac{1}{2}(A+B+2 C) u_{2,122} \\
& \tau_{1}=\tau_{1}^{Q}=B u_{1,11}+\frac{1}{2}(A+B-2 C) u_{1,22}+2 D u_{2,12} \\
& \tau_{2}=\tau_{2}^{Q}=(B-A-2 D) u_{1,12}+A u_{2,11}+\frac{1}{2}(A+B-2 C) u_{2,22}
\end{aligned}
$$

We remark that $t_{1}^{Q}$ in (21) and $t_{2}^{Q}$ in (22) are the opposite of $t_{1}^{S}$ in (17) and of $t_{2}^{S}$ in (18), respectively, and that $\tau_{1}^{Q}$ in (23) and $\tau_{2}^{Q}$ in (24) are the same of $\tau_{1}^{S}$ in (19) and of $\tau_{2}^{S}$ in (20), respectively. have

The characterization of side $R$ is done by setting $n_{i}=\delta_{i 2}$. Thus, from (15) with $\alpha=1,2$, and from (16) with $\alpha=1,2$, we

$$
\begin{aligned}
& t_{1}=t_{1}^{R}=\mu\left(u_{1,2}+u_{2,1}\right)-(B-A) u_{2,122}-(B-A-2 D) u_{2,111}-A u_{1,222}-\frac{1}{2}(A+B+2 C) u_{1,112} \\
& t_{2}=t_{2}^{R}=u_{2,2}(\lambda+2 \mu)+u_{1,1} \lambda-B u_{2,222}-2 D u_{1,111}-\frac{1}{2}(A+B+2 C) u_{2,112}-(B-A) u_{1,122} \\
& \tau_{1}=\tau_{1}^{R}=(B-A-2 D) u_{2,12}+A u_{1,22}+\frac{1}{2}(A+B-2 C) u_{1,11} \\
& \tau_{2}=\tau_{2}^{R}=B u_{2,22}+\frac{1}{2}(A+B-2 C) u_{2,11}+2 D u_{1,12}
\end{aligned}
$$

We remark that, because of isotropy, $t_{1}^{R}$ in (25) and $t_{2}^{R}$ in (26) are the same of $t_{2}^{S}$ in (18) and of $t_{1}^{S}$ in (17), respectively, by changing the indexes 1 and 2. Besides, because of isotropy, $\tau_{1}^{R}$ in (27) and $\tau_{2}^{R}$ in (28) are the same of $\tau_{2}^{S}$ in (19) and of $\tau_{1}^{S}$ in (20), respectively, by changing the indexes 1 and 2 .

Finally, the characterization of side $T$ is done by setting $n_{i}=-\delta_{i 2}$. Thus, from (15) with $\alpha=1,2$, and from (16) with $\alpha=1,2$, we have

$$
t_{1}=t_{1}^{T}=-\mu\left(u_{1,2}+u_{2,1}\right)+(B-A) u_{2,122}+(B-A-2 D) u_{2,111}+A u_{1,222}+\frac{1}{2}(A+B+2 C) u_{1,112}
$$




$$
\begin{aligned}
& t_{2}=t_{2}^{T}=-u_{2,2}(\lambda+2 \mu)-u_{1,1} \lambda+B u_{2,222}+2 D u_{1,111}+\frac{1}{2}(A+B+2 C) u_{2,112}+(B-A) u_{1,122}, \\
& \tau_{1}=\tau_{1}^{T}=(B-A-2 D) u_{2,12}+A u_{1,22}+\frac{1}{2}(A+B-2 C) u_{1,11}, \\
& \tau_{2}=\tau_{2}^{T}=B u_{2,22}+\frac{1}{2}(A+B-2 C) u_{2,11}+2 D u_{1,12} .
\end{aligned}
$$

We remark that $t_{1}^{T}$ in (29) and $t_{2}^{T}$ in (30) are the opposite of $t_{1}^{R}$ in (25) and of $t_{2}^{R}$ in (26), respectively, and that $\tau_{1}^{T}$ in (31) and $\tau_{2}^{T}$ in (32) are the same of $\tau_{1}^{R}$ in (27) and of $\tau_{2}^{R}$ in (28), respectively.

\subsubsection{Characterization of vertices}

The last equation of $(8)$ is reduced, because of $(9)_{2}$ to

$$
\begin{aligned}
& \int_{[\partial \partial \mathcal{B}]} \delta u_{\alpha}\left(f_{\alpha}-f_{\alpha}^{e x t}\right) \\
& =\left[\delta u_{\alpha}\left(T_{\alpha i j} V_{i j}-f_{\alpha}^{e x t}\right)\right]_{\mathcal{V}_{1}}+\left[\delta u_{\alpha}\left(T_{\alpha i j} V_{i j}-f_{\alpha}^{e x t}\right)\right]_{\mathcal{V}_{2}} \\
& \quad+\left[\delta u_{\alpha}\left(T_{\alpha i j} V_{i j}-f_{\alpha}^{e x t}\right)\right]_{\mathcal{V}_{3}}+\left[\delta u_{\alpha}\left(T_{\alpha i j} V_{i j}-f_{\alpha}^{e x t}\right)\right]_{\mathcal{V}_{4}},
\end{aligned}
$$

For vertex $\mathcal{V}_{1}$ the side $A$ has $n_{j}=-\delta_{1 j}$ and $v_{i}=\delta_{i 2}$ and the side $B$ has $n_{j}=\delta_{2 j}$ and $v_{i}=-\delta_{i 1}$ so that

$$
\left[V_{i j}\right]_{\mathcal{v}_{1}}=\left[v_{i}^{l} n_{j}^{l}+v_{i}^{r} n_{j}^{r}\right]_{\mathcal{V}_{1}}=-\delta_{i 2} \delta_{1 j}-\delta_{i 1} \delta_{2 j}
$$

For vertex $\mathcal{V}_{2}$ the side $B$ has $n_{j}=\delta_{2 j}$ and $v_{i}=\delta_{i 1}$ and the side $C$ has $n_{j}=\delta_{1 j}$ and $v_{i}=\delta_{i 2}$ so that

$$
\left[V_{i j}\right]_{\mathcal{V}_{2}}=\left[v_{i}^{l} n_{j}^{l}+v_{i}^{r} n_{j}^{r}\right]_{\mathcal{V}_{2}}=\delta_{i 1} \delta_{2 j}+\delta_{i 2} \delta_{1 j}
$$

For vertex $v_{3}$ the side $C$ has $n_{j}=\delta_{1 j}$ and $v_{i}=-\delta_{i 2}$ and the side $D$ has $n_{j}=-\delta_{2 j}$ and $v_{i}=\delta_{i 1}$ so that

$$
\left[V_{i j}\right]_{\mathcal{V}_{3}}=\left[v_{i}^{l} n_{j}^{l}+v_{i}^{r} n_{j}^{r}\right]_{\mathcal{V}_{3}}=-\delta_{i 2} \delta_{1 j}-\delta_{i 1} \delta_{2 j}
$$

For vertex $v_{4}$ the side $D$ has $n_{j}=-\delta_{2 j}$ and $v_{i}=-\delta_{i 1}$ and the side $A$ has $n_{j}=-\delta_{1 j}$ and $v_{i}=-\delta_{i 2}$ so that

$$
\left[V_{i j}\right]_{\mathcal{V}_{4}}=\left[v_{i}^{l} n_{j}^{l}+v_{i}^{r} n_{j}^{r}\right]_{\mathcal{V}_{4}}=\delta_{i 1} \delta_{2 j}+\delta_{i 2} \delta_{1 j}
$$

Thus, finally, the (33) yields

$$
\begin{aligned}
& \int_{[\partial \partial \mathcal{B}]} \delta u_{\alpha}\left(f_{\alpha}-f_{\alpha}^{e x t}\right)=\left[\delta u_{\alpha}\left(-T_{\alpha 21}-T_{\alpha 12}-f_{\alpha}^{e x t}\right)\right]_{\mathcal{V}_{1}}+\left[\delta u_{\alpha}\left(T_{\alpha 12}+T_{\alpha 21}-f_{\alpha}^{e x t}\right)\right]_{\mathcal{V}_{2}} \\
& \quad+\left[\delta u_{\alpha}\left(-T_{\alpha 21}-T_{\alpha 12}-f_{\alpha}^{e x t}\right)\right]_{\mathcal{V}_{3}}+\left[\delta u_{\alpha}\left(T_{\alpha 12}+T_{\alpha 21}-f_{\alpha}^{e x t}\right)\right]_{\mathcal{V}_{4}},
\end{aligned}
$$

where $T_{\alpha 12}+T_{\alpha 21}$, in terms of the displacement field, it is for $\alpha=1$

$$
T_{112}+T_{121}=2 C u_{1,12}+(B-A-2 D) u_{2,11}+2 D u_{2,22},
$$

and for $\alpha=2$,

$$
T_{212}+T_{221}=2 C u_{2,12}+(B-A-2 D) u_{1,22}+2 D u_{1,11} .
$$

\section{Numerical simulations}

Numerical data for the simulations that will be presented in this paper are here shown (see Fig. 1)

$$
\begin{aligned}
& L=2 m, l=1 m, \mu=10 M P a m, \lambda=15 \text { MPa } m, \rho=10^{5} \mathrm{Kg} / \mathrm{m}^{2} E=\frac{\mu(3 \lambda+2 \mu)}{\lambda+\mu}=26 \text { MPa m, } \\
& \alpha_{1}=E l_{m}^{2}, \quad \alpha_{2}=E l_{m}^{2}, \quad \alpha_{3}=2 E l_{m}^{2}, \quad \alpha_{4}=E l_{m}^{2}, \quad \alpha_{5}=\frac{1}{2} E l_{m}^{2}, \quad l_{m}=10 \mathrm{~cm},
\end{aligned}
$$

and therefore

$$
A=7 E l_{m}^{2}, \quad B=34 E l_{m}^{2}, \quad C=\frac{21}{2} E l_{m}^{2}, \quad D=8 E l_{m}^{2} .
$$

With these data the positive definiteness of the strain energy functional is guaranteed. 


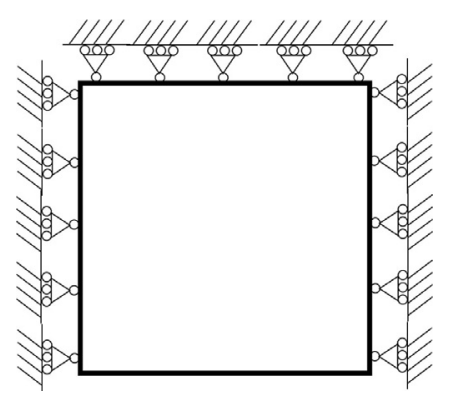

Fig. 2. Graphical representation of the kinematic restrictions for the heavy sheet problem.

\subsection{The heavy sheet problem}

We consider an heavy sheet appended at the top side $R$ and constrained at sides $Q$ and $S$ to have null horizontal displacement. Thus, the kinematical restrictions are

$$
\left(\delta u_{2}\right)_{R}=0, \quad\left(\delta u_{1}\right)_{Q}=0, \quad\left(\delta u_{1}\right)_{S}=0 .
$$

and also represented in Fig. 2. Let the two partial differential Eqs. (6) and (7) be satisfied with the following external forces per unit area,

$$
b_{1}^{e x t}=0, \quad b_{2}^{e x t}=-\rho g,
$$

and let the edge boundary conditions be as follows,

$$
\begin{aligned}
& u_{1}=0, \quad t_{2}^{Q}=0, \quad \tau_{1}^{Q}=0, \quad \tau_{2}^{Q}=\tau_{2}^{e x t, Q}, \quad \forall X \in Q, \\
& t_{1}^{R}=0, \quad u_{2}=0, \quad \tau_{1}^{R}=0, \quad \tau_{2}^{R}=\tau_{2}^{R, e x t}, \quad \forall X \in R, \\
& u_{1}=0, \quad t_{2}^{S}=0, \quad \tau_{1}^{S}=0, \quad \tau_{2}^{S}=\tau_{2}^{e x t, S}, \quad \forall X \in S, \\
& t_{1}^{T}=0, \quad t_{2}^{T}=0, \quad \tau_{1}^{T}=0, \quad \tau_{2}^{T}=\tau_{2}^{T, e x t}, \quad \forall X \in T,
\end{aligned}
$$

where the non-null external edge double forces are as follows,

$$
\tau_{2}^{\text {ext }, Q}=\tau_{2}^{\text {ext }, S}=\frac{(A+B-2 C) \rho g}{2(\lambda+2 \mu)}, \quad \tau_{2}^{R, e x t}=\tau_{2}^{T, e x t}=\frac{\rho g B}{(\lambda+2 \mu)},
$$

and let the only wedge conditions that are not implied by (41)-(44) be as follows,

$$
\left(f_{2}^{e x t}\right)_{V_{3}}=0, \quad\left(f_{2}^{e x t}\right)_{V_{4}}=0 \text {. }
$$
field,

The analytical solution of this problem is achieved in Luca et al. (2015) and here represented in terms of the displacement

$$
u_{1}=0, \quad u_{2}=\frac{\rho g\left(X_{2}-l\right)\left(3 l+X_{2}\right)}{2(\lambda+2 \mu)} .
$$

The numerical simulations of this problem is shown in Fig. 3a and have shown remarkable identification between exact analytical solution and the respective numerical simulation (Fig. 3b)

\subsection{The bending problem}

We consider the bending problem and constrain the whole side $A$ to not displace in the horizontal direction and one of its point, the origin $O$, to have also null vertical displacement. Thus, the kinematical restrictions are

$$
\left(\delta u_{1}\right)_{A}=0, \quad\left(\delta u_{2}\right)_{O}=0 .
$$

and they are represented in Fig. 4. It has to be remarked that such kinematical constrains are not of a general type. In fact, the $(48)_{2}$ is referred to a single point that is not a vertex of the domain. This means that the results will be reasonable only in the case of vertical null force at point 0 . Let the two partial differential Eqs. (6) and (7) be satisfied with the null external forces per unit area,

$$
b_{1}^{e x t}=0, \quad b_{2}^{e x t}=0
$$

and the edge boundary conditions be as follows, 

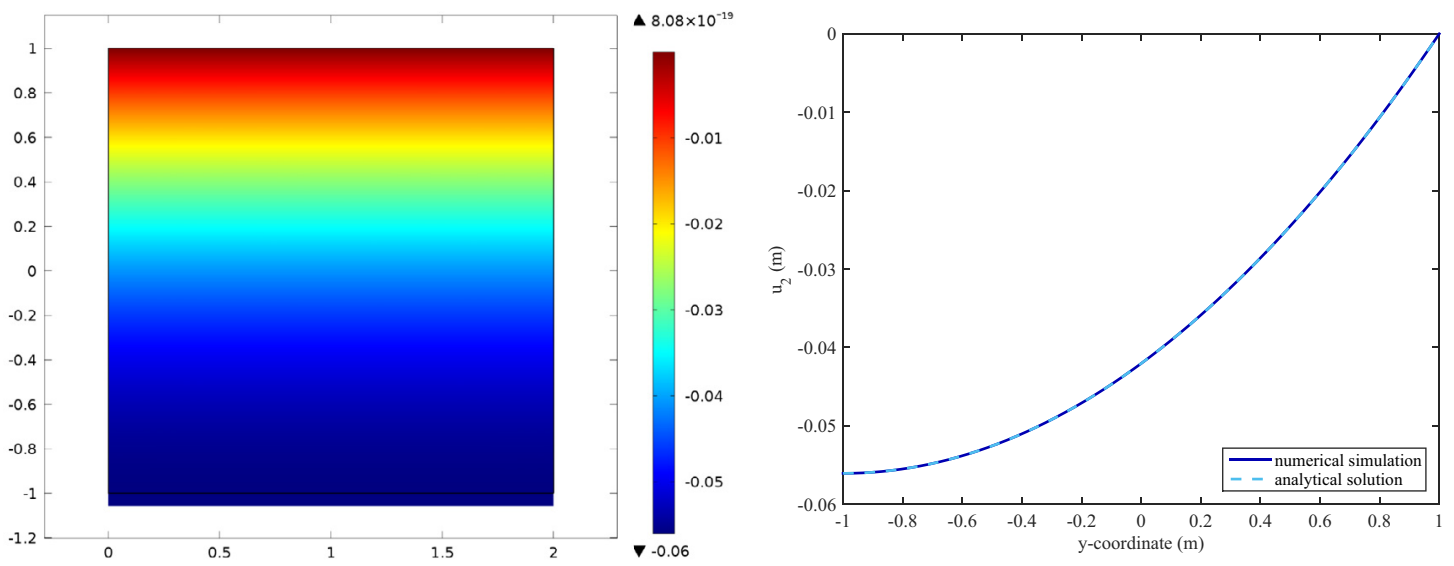

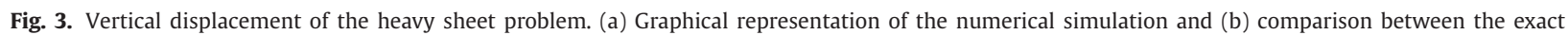
analytical solution and the respective numerical one through a given vertical cut.

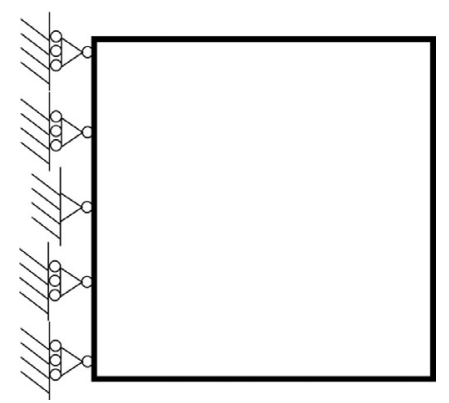

Fig. 4. Graphical representation of the kinematic restrictions for the bending problem.

$$
\begin{aligned}
& u_{1}=0, \quad t_{2}^{Q}=0, \quad \tau_{1}^{Q}=0, \quad \tau_{2}^{Q}=\tau_{2}^{\text {ext }, Q}, \quad \forall X \in Q, \\
& t_{1}^{R}=0, \quad t_{2}^{R}=0, \quad \tau_{1}^{R}=0, \quad \tau_{2}^{R}=\tau_{2}^{R, \text { ext }}, \quad \forall X \in R, \\
& t_{1}=t_{1}^{\text {ext }, S}, \quad t_{2}^{S}=0, \quad \tau_{1}^{S}=0, \quad \tau_{2}^{S}=\tau_{2}^{\text {ext }, S}, \quad \forall X \in S, \\
& t_{1}^{T}=0, \quad t_{2}^{T}=0, \quad \tau_{1}^{T}=0, \quad \tau_{2}^{T}=\tau_{2}^{T, \text { ext }}, \quad \forall X \in T,
\end{aligned}
$$

where the non-null external edge force and double forces are as follows,

$$
\begin{aligned}
& t_{1}^{\text {ext }, S}=\frac{3 M^{\text {ext }} X_{2}}{2 l^{3}}, \\
& \tau_{2}^{\text {ext }, Q}=\tau_{2}^{\text {ext }, S}=\frac{3 M^{\text {ext }}[-(5 \lambda+8 \mu) A+(\lambda+4 \mu) B+2 \lambda C-(4 \lambda+8 \mu) D]}{16 l^{3} \mu(\lambda+\mu)}, \\
& \tau_{2}^{R, e x t}=\tau_{2}^{T, \text { ext }}=-\frac{3 M^{\text {ext }}[(\lambda+2 \mu) A+(3 \lambda+2 \mu) B-(2 \lambda+4 \mu) C-(4 \lambda+8 \mu) D]}{16 l^{3} \mu(\lambda+\mu)} .
\end{aligned}
$$

and let the only wedge conditions that are not implied by (50)-(53) be as follows,

$$
\begin{aligned}
& \left(f_{2}^{\text {ext }}\right)_{V_{1}}=0, \quad\left(f_{2}^{\text {ext }}\right)_{V_{2}}=0, \quad\left(f_{2}^{\text {ext }}\right)_{V_{3}}=0, \quad\left(f_{2}^{\text {ext }}\right)_{V_{4}}=0, \\
& \left(f_{1}^{\text {ext }}\right)_{V_{2}}=-\left(f_{1}^{\text {ext }}\right)_{V_{3}}=\frac{3 M^{\text {ext }}[(\lambda+2 \mu)(A-B+2 C)+4 \mu D]}{8 l^{3} \mu(\lambda+\mu)} .
\end{aligned}
$$
field,

The analytical solution of this problem is achieved in Luca et al. (2015) and here represented in terms of the displacement

$$
u_{1}=\frac{3 M^{\text {ext }}(\lambda+2 \mu) X_{1} X_{2}}{8 l^{3} \mu(\lambda+\mu)}, \quad u_{2}=-\frac{3 M^{\text {ext }}\left[\lambda X_{2}^{2}+(\lambda+2 \mu) X_{1}^{2}\right]}{16 l^{3} \mu(\lambda+\mu)},
$$



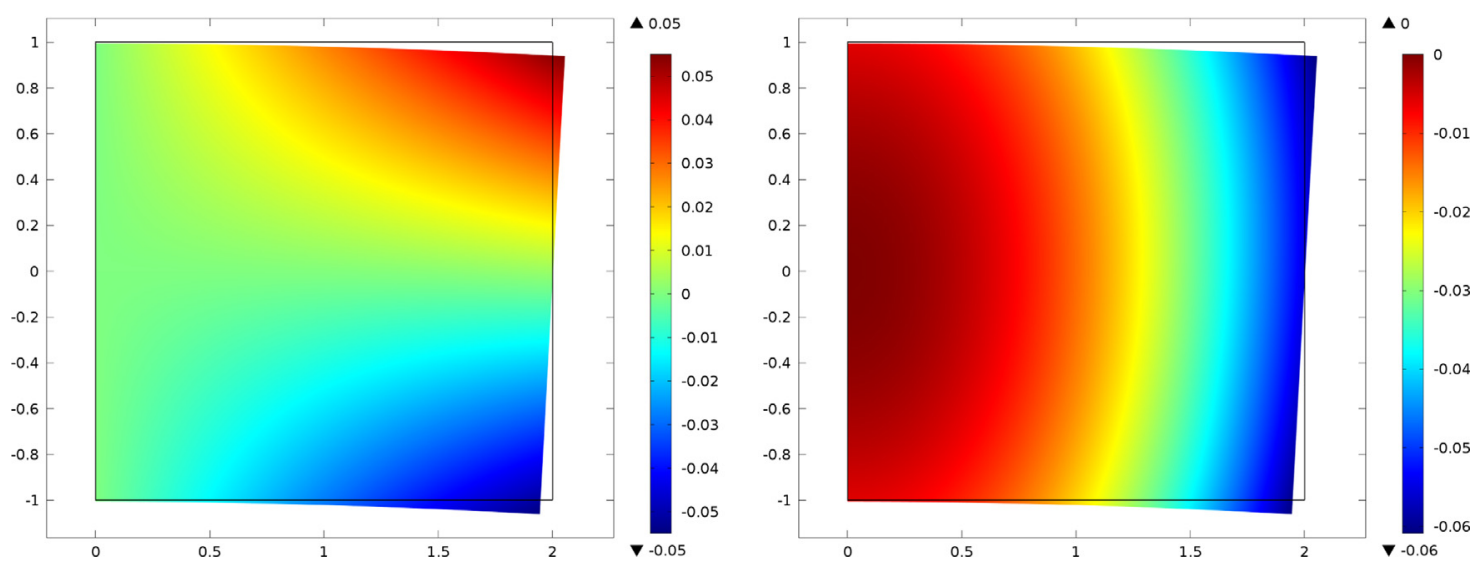

Fig. 5. Numerical simulation for the bending problem. In particular, (a) horizontal and (b) vertical displacements are represented.

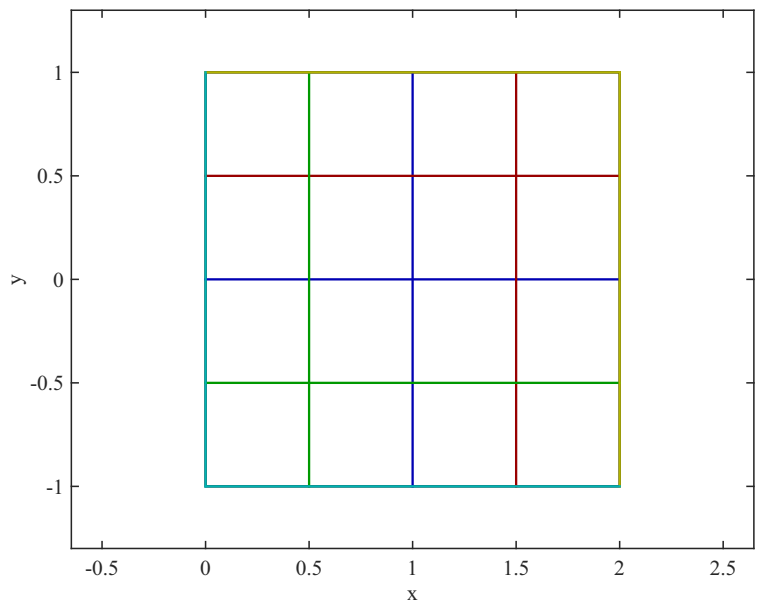

Fig. 6. Horizontal and vertical cuts that are used to represent the comparison between the exact analytical and numerical simulations in Figs $7-10$, 13 and 14. In such figures the colors of the lines correspond to the colors of the cuts represented in this figure. (For interpretation of the references to colour in this figure legend, the reader is referred to the web version of this article.)

The numerical simulations of this problem are shown in Figs. 5.

The comparisons between the exact analytical solution and the numerical simulation have been done through the horizontal and vertical cuts of Fig. 6 and the respective numerical simulation is shown in Figs. 7 and 8.

\subsection{The bending problem without double forces}

We consider the same bending problem of the previous subsection, with the same kinematical restrictions (48), the same external forces per unit area (49), the same edge boundary conditions (50)-(53) with the same edge force (54) but with null edge double forces

$$
\tau_{2}^{e x t, Q}=\tau_{2}^{e x t, S}=0, \quad \tau_{2}^{R, e x t}=\tau_{2}^{T, e x t}=0,
$$

and null wedge conditions

$$
\left(f_{2}^{e x t}\right)_{V_{1}}=0, \quad\left(f_{2}^{e x t}\right)_{V_{2}}=0, \quad\left(f_{2}^{e x t}\right)_{V_{3}}=0, \quad\left(f_{2}^{\text {ext }}\right)_{V_{4}}=0,\left(f_{1}^{\text {ext }}\right)_{V_{2}}=-\left(f_{1}^{\text {ext }}\right)_{V_{3}}=0 .
$$

In this case we do not have an analytical solution but we make numerical simulations, that are shown in Figs. 9 and 10.

It can be remarked that the presence of double forces has a relatively strong influence on the numerical results.

\subsection{The flexure problem}

We consider the flexure problem and constrain the whole side $C$ to displace in the vertical direction and one of the point of side $A$, the origin $O$, to have null horizontal and vertical displacements. Thus, the kinematical restrictions are

$$
\left(\delta u_{1}\right)_{S}=0, \quad\left(\delta u_{1}\right)_{O}=0 \quad\left(\delta u_{2}\right)_{O}=0 .
$$



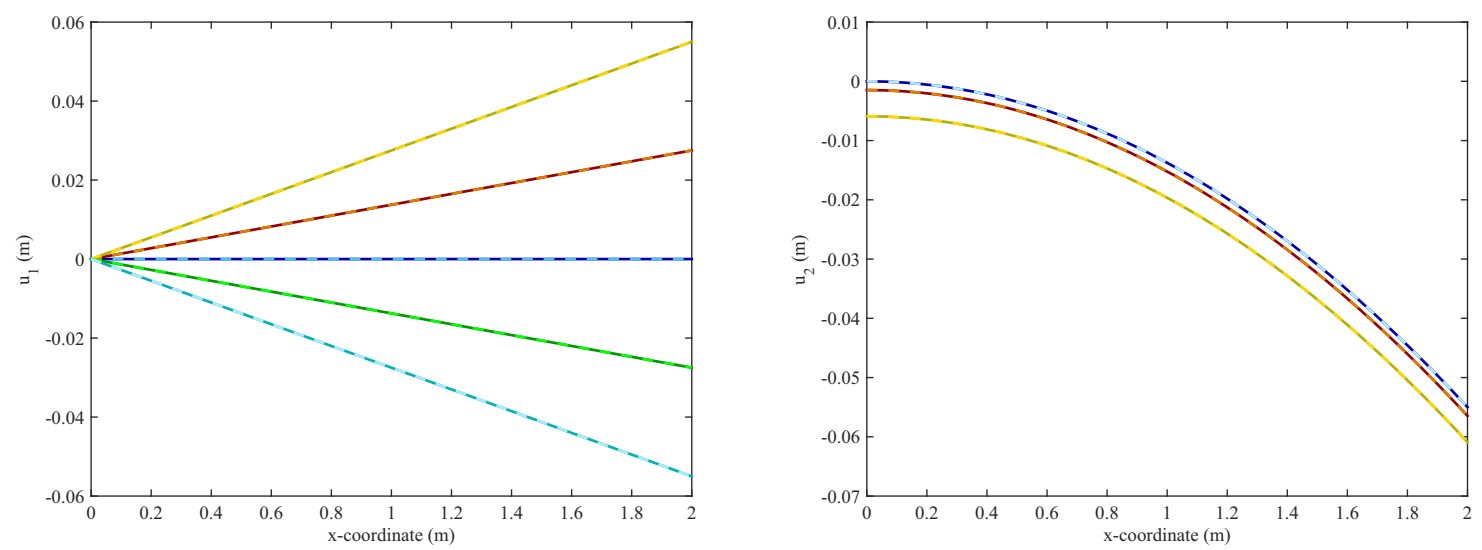

Fig. 7. Comparison between the exact analytical solution and numerical simulation through the horizontal cuts of Fig. 6. In particular, (a) horizontal and (b) vertical displacements are represented.
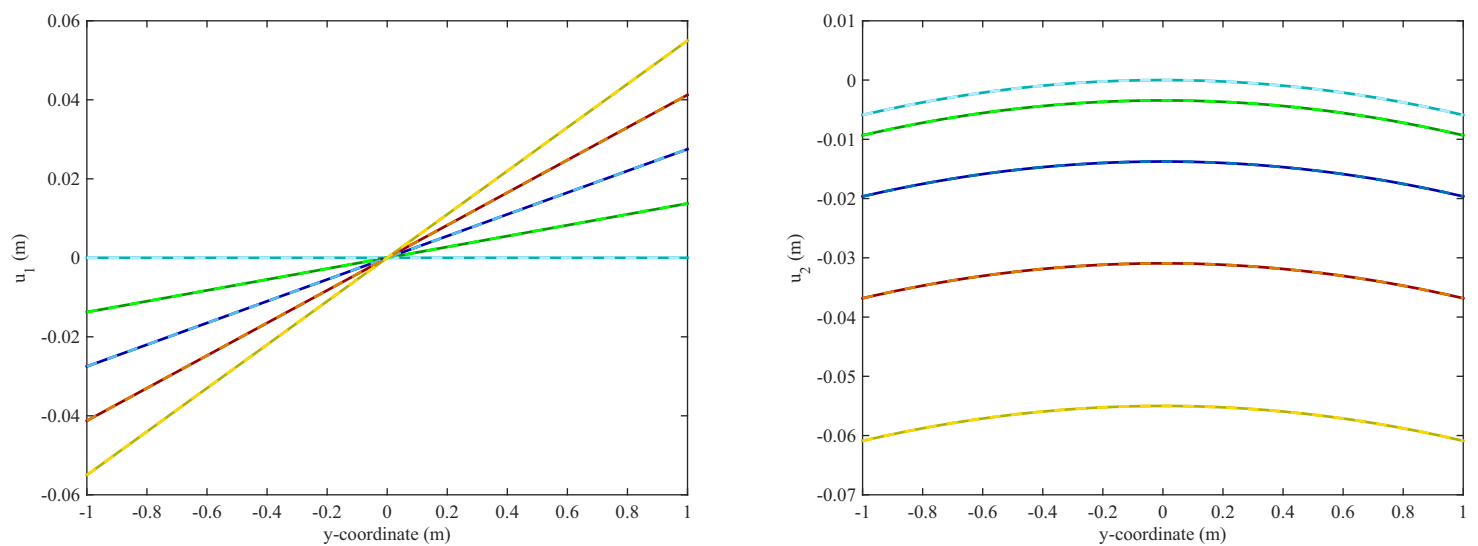

Fig. 8. Comparison between the exact analytical solution and numerical simulation through the vertical cuts of Fig. 6. In particular, (a) horizontal and (b) vertical displacements are represented.

曰
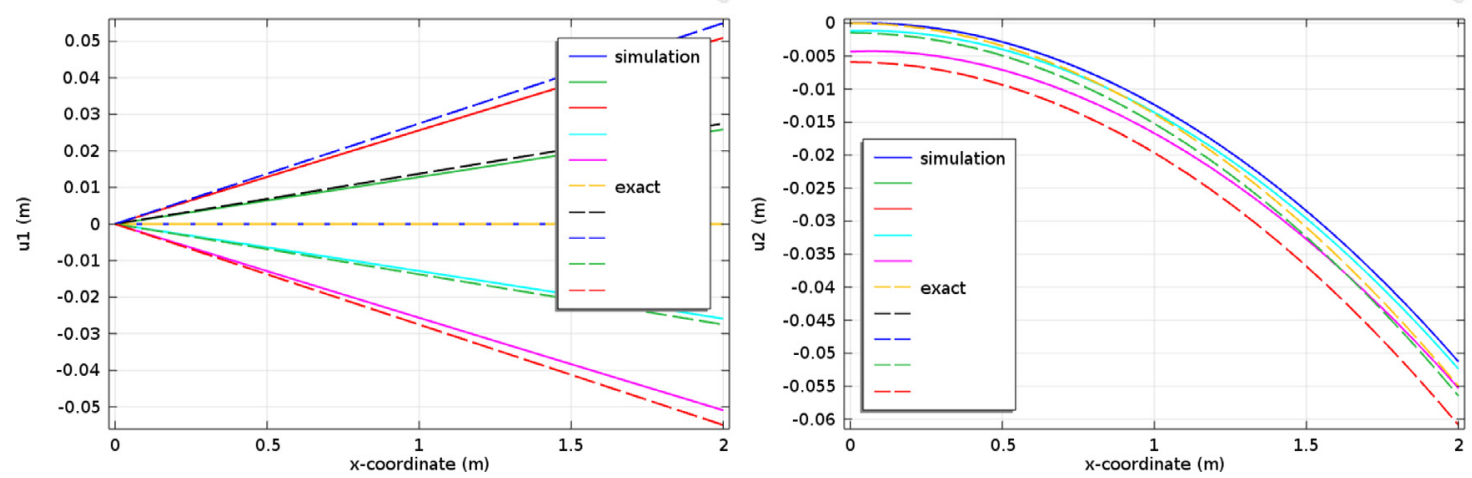

Fig. 9. Comparison between the exact analytical solution with non null external double and wedge forces and numerical simulation with null external double and wedge forces through the horizontal cuts of Fig. 6. In particular, (a) horizontal and (b) vertical displacements are represented.

and they are represented in Fig. 11. It has to be remarked that such kinematical constrain are not of a general type. In fact, the $(58)_{2,3}$ is referred to a single point that is not a vertex of the domain. This means that the results will be reasonable only in the case of null force at point 0 . Let the two partial differential Eqs. (6) and (7) be satisfied with null external forces per unit area (49), let the edge boundary conditions be as follows,

$$
t_{1}=t_{1}^{\text {ext,Q }}, \quad t_{2}^{Q}=t_{2}^{\text {ext,Q }}, \quad \tau_{1}^{Q}=\tau_{1}^{\text {ext,Q }}, \quad \tau_{2}^{Q}=\tau_{2}^{\text {ext,Q }}, \quad \forall X \in Q,
$$



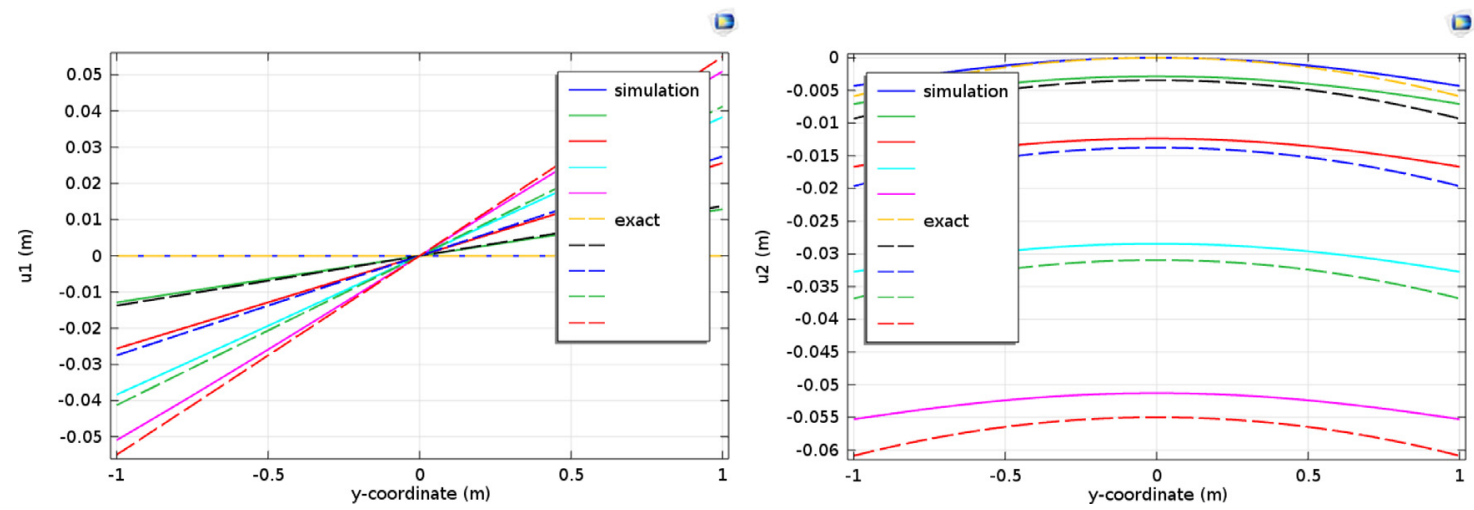

Fig. 10. Comparison between the exact analytical solution with non null external double and wedge forces and numerical simulation with null external double and wedge forces through the vertical cuts of Fig. 6. In particular, (a) horizontal and (b) vertical displacements are represented.

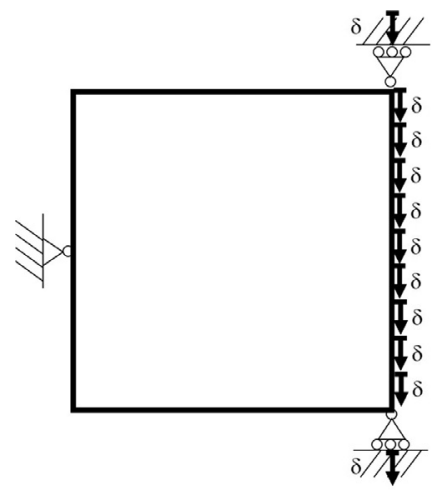

Fig. 11. Graphical representation of the kinematic restrictions for the flexure problem.

$$
\begin{aligned}
& t_{1}=t_{1}^{\text {ext,R}}, \quad t_{2}^{R}=0, \quad \tau_{1}^{R}=\tau_{1}^{\text {ext, }, R}, \quad \tau_{2}^{R}=\tau_{2}^{R, e x t}, \quad \forall X \in R, \\
& t_{1}=0, \quad u_{2}=-\delta, \quad \tau_{1}^{S}=\tau_{1}^{\text {ext }, S}, \quad \tau_{2}^{S}=0, \quad \forall X \in S, \\
& t_{1}=t_{1}^{\text {ext }, T}, \quad t_{2}^{T}=0, \quad \tau_{1}^{T}=\tau_{1}^{\text {ext }, T}, \quad \tau_{2}^{T}=\tau_{2}^{T, e x t}, \quad \forall X \in T,
\end{aligned}
$$

where the non-null external edge force and double forces are as follows,

$$
\begin{aligned}
t_{1}^{e x t, Q} & =-\frac{3 L Q X_{2}}{2 l^{3}} \\
t_{2}^{\text {ext }, Q} & =\frac{3 Q\left[-A \lambda+B(5 \lambda+4 \mu)+2 C \lambda-4 D(3 \lambda+4 \mu)+4 \mu \lambda\left(l^{2}-X_{2}^{2}\right)+4 \mu^{2}\left(l^{2}-X_{2}^{2}\right)\right]}{16 l^{3} \mu(\lambda+\mu)} \\
t_{1}^{e x t, R} & =-t_{1}^{\text {ext }, T}=-\frac{3 Q}{16 l^{3} \mu(\lambda+\mu)}[(\lambda+2 \mu)(A-2 C-4 D)+B(3 \lambda+2 \mu)] \\
t_{1}^{\text {ext }, S} & =\frac{3 M^{\text {ext }} X_{2}}{2 l^{3}}, \\
\tau_{1}^{\text {ext }, Q} & =\tau_{1}^{\text {ext }, S}=\frac{3 Q X_{2}[(3 \lambda+4 \mu)(A-2 C)+\lambda(B+4 D)]}{16 l^{3} \mu(\lambda+\mu)} \\
\tau_{2}^{\text {ext }, Q} & =-\frac{3 Q L[(5 \lambda+8 \mu) A-(\lambda+4 \mu) B-2 \lambda C+(\lambda+2 \mu) 4 D]}{16 l^{3} \mu(\lambda+\mu)} \\
\tau_{1}^{\text {ext }, R} & =-\tau_{1}^{\text {ext }, T}=\frac{3 Q[(\lambda+2 \mu)(3 A+2 C)+(\lambda-2 \mu) B-4 \lambda D]}{16 l^{2} \mu(\lambda+\mu)} \\
\tau_{2}^{\text {ext }, R} & =\tau_{2}^{\text {ext }, T}=-\frac{3 Q\left(L-X_{1}\right)[(\lambda+2 \mu)(A-2 C-4 D)+(3 \lambda+2 \mu) B]}{16 l^{3} \mu(\lambda+\mu)}
\end{aligned}
$$



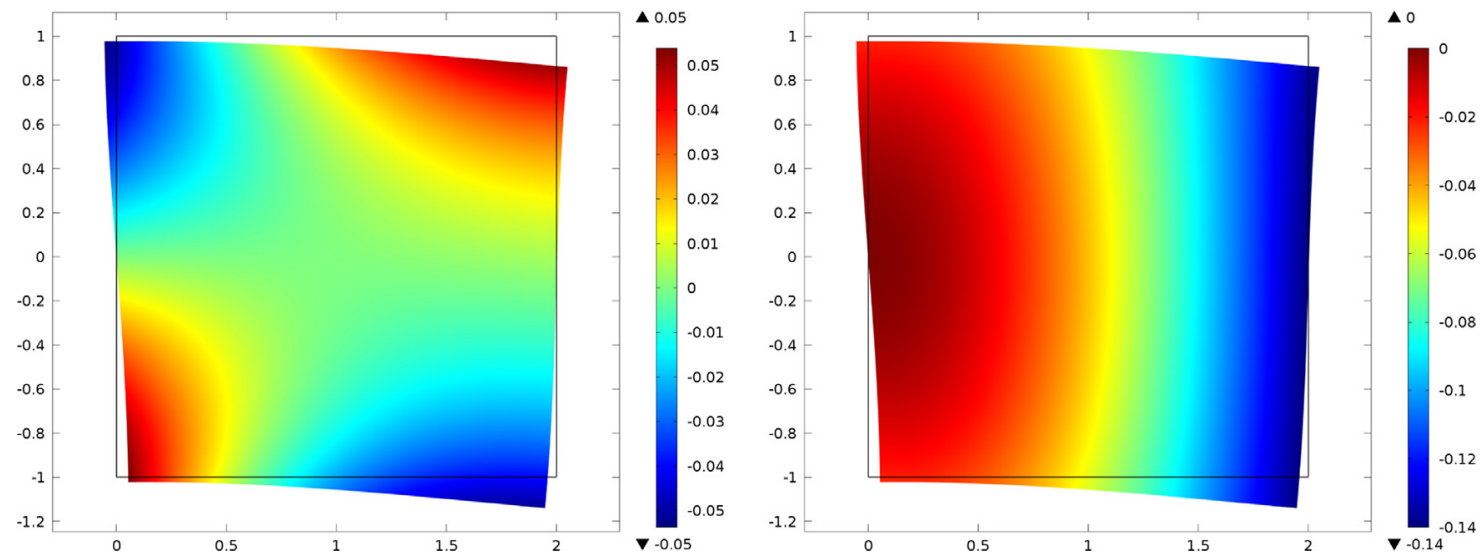

Fig. 12. Numerical simulation for the flexure problem. In particular, (a) horizontal and (b) vertical displacements are represented.

口
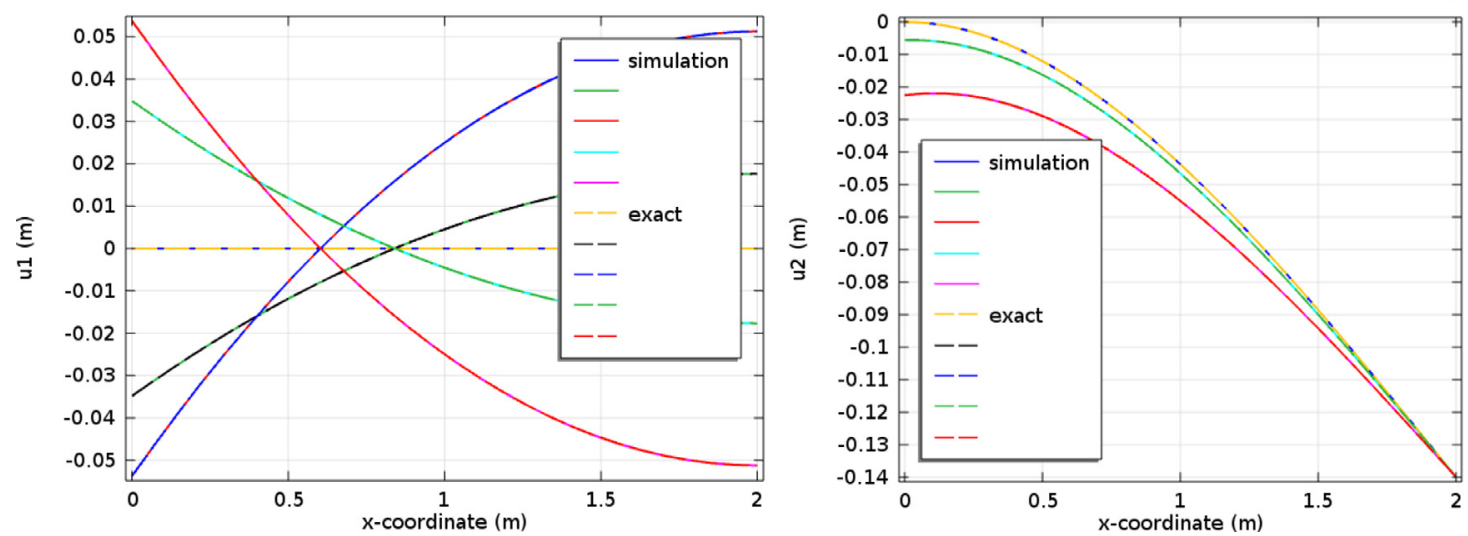

Fig. 13. Comparison between the exact analytical solution and numerical simulation through the horizontal cuts of Fig. 6. In particular, (a) horizontal and (b) vertical displacements are represented.

and the only wedge conditions that are not implied by (59)-(62) are as follows,

$$
\begin{aligned}
& \left(f_{1}^{e x t}\right)_{V_{1}}=-\left(f_{1}^{e x t}\right)_{V_{4}}=-\frac{3 Q L[(\lambda+2 \mu)(A-B+2 C)+4 \mu D]}{8 l^{3} \mu(\lambda+\mu)} \\
& \left(f_{2}^{\text {ext }}\right)_{V_{1}}=\left(f_{2}^{e x t}\right)_{V_{4}}=\frac{3 Q[(3 \lambda+4 \mu)(A-B)-2 \lambda C+4(2 \lambda+3 \mu) D]}{8 l^{2} \mu(\lambda+\mu)} \\
& \left(f_{1}^{\text {ext }}\right)_{V_{2}}=0, \quad\left(f_{1}^{e x t}\right)_{V_{3}}=0 .
\end{aligned}
$$

The analytical solution of this problem is achieved in Luca et al. (2015) and here represented in terms of the displacement field,

$$
\begin{aligned}
& u_{1}=-\frac{Q X_{2}\left[(\lambda+2 \mu)\left(3 X_{1}^{2}-X_{2}^{2}-6 L X_{1}\right)+2(\lambda+\mu)\left(6 l^{2}-X_{2}^{2}\right)\right]}{16 l^{3} \mu(\lambda+\mu)}, \\
& u_{2}=-\frac{Q\left[\left(3 L-X_{1}\right)(\lambda+2 \mu) X_{1}^{2}+3\left(L-X_{1}\right) \lambda X_{2}^{2}\right]}{16 l^{3} \mu(\lambda+\mu)},
\end{aligned}
$$

The numerical simulations of this problem are shown in Figs. 12.

The comparisons between the exact analytical solution and the numerical simulation have been done through the horizontal and vertical cuts of Fig. 6 and the respective numerical simulation is shown in Figs. 13 and 14 .

\subsection{Convergence analysis for the wedge force problem}

We consider again the bending problem with the same kinematical restrictions (48) (see also Fig. 4), the same external forces per unit area (49), the same edge boundary conditions (50)-(53) but with null edge forces and double forces at side 

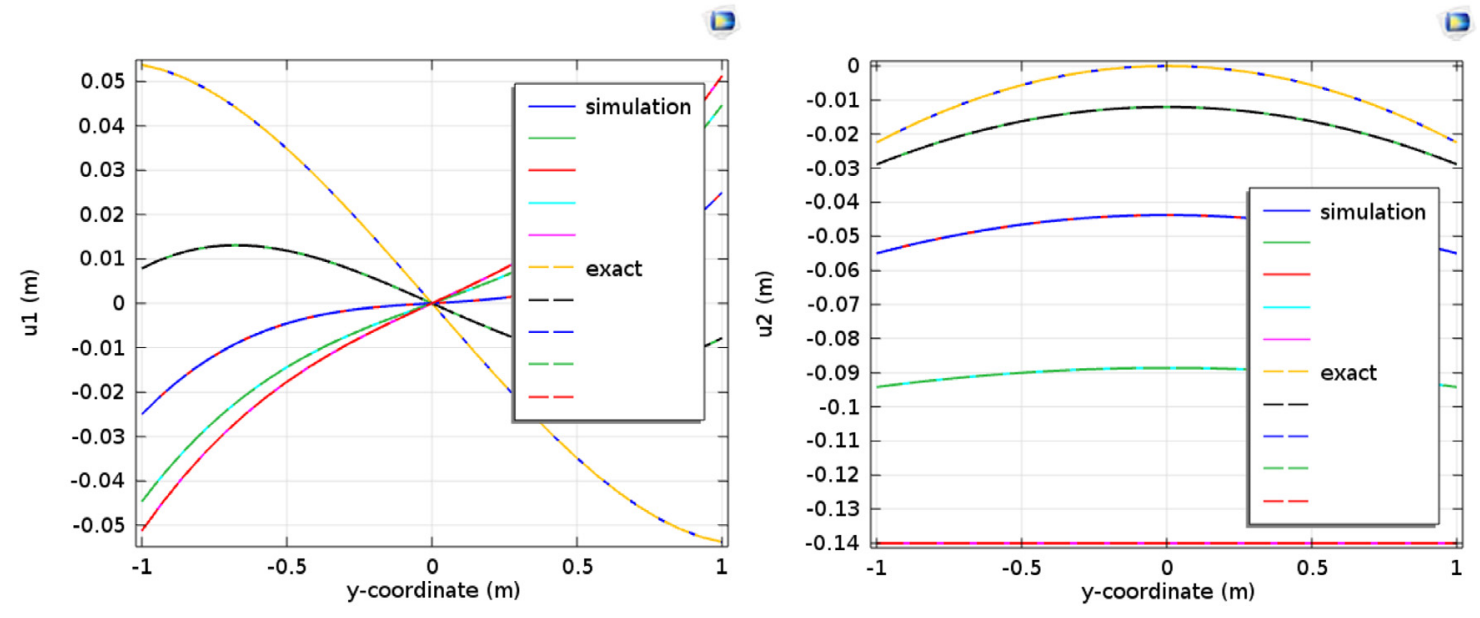

Fig. 14. Comparison between the exact analytical solution and numerical simulation through the vertical cuts of Fig. 6. In particular, (a) horizontal and (b) vertical displacements are represented.

s_mesh $(30)=0.02$ Surface: Dependent variable u2 $(\mathrm{m})$

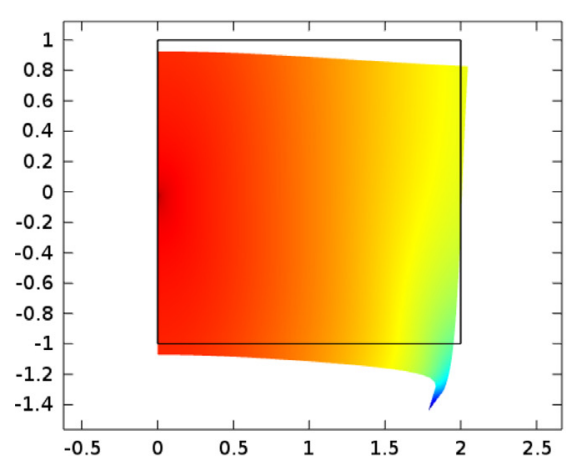

D

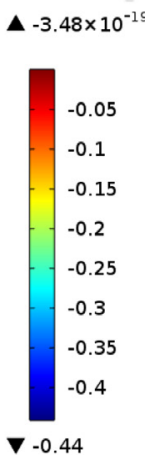

s_mesh(30) $=0.02$ Surface: Dependent variable u2 $(\mathrm{m})$

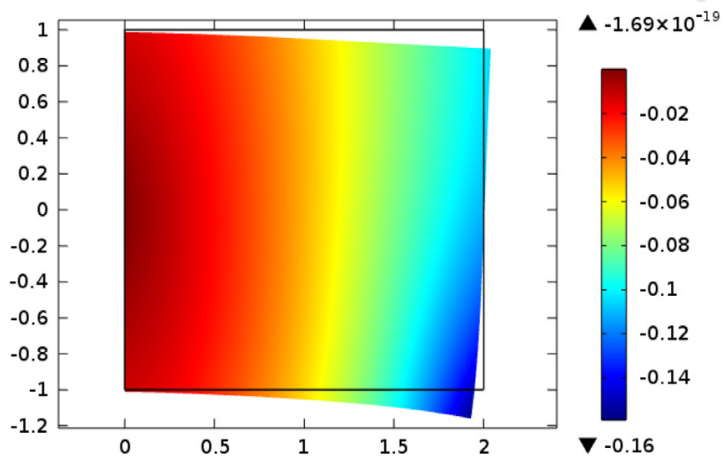

Fig. 15. Comparison between the vertical displacement in the (a) first and (b) second gradient models in the case of an external wedge force.

$C$ as follows,

$$
t_{1}^{e x t, S}=0, \quad \tau_{2}^{e x t, Q}=\tau_{2}^{e x t, S}=0, \quad \tau_{2}^{R, e x t}=\tau_{2}^{T, e x t}=0,
$$

and with the following system of wedge conditions

$$
\left(f_{2}^{e x t}\right)_{V_{1}}=0, \quad\left(f_{2}^{e x t}\right)_{V_{2}}=0, \quad\left(f_{2}^{e x t}\right)_{V_{3}}=-F, \quad\left(f_{2}^{e x t}\right)_{V_{4}}=0, \quad\left(f_{1}^{e x t}\right)_{V_{2}}=-\left(f_{1}^{\text {ext }}\right)_{V_{3}}=0 .
$$

Even in this modified bending case we do not have an analytical solution but we make numerical simulations, that are shown in Figs 15. On the left-hand side we show the results of a numerical simulation of the analogous problem in the classical first gradient model and on the right-hand side a the results of a numerical simulation with the present second gradient model.

It is immediately visible from Fig. 15 that the first gradient model is not adequate for wedge concentrated external forces. In Fig. 16 a convergence analysis is performed. From such a convergence analysis we deduce that first gradient models are not adequate to model concentrated external forces.

\section{Experimental evidence of elastic second gradient contribution to the deformation energy}

In Ferretti et al. (2014) it is shown that second-gradient energy terms allow the onset of internal shear boundary layers. These boundary layers are transition zones between two different shear deformation modes. In the same paper, on the one hand it is claimed that their existence cannot be described by a simple first-gradient model, and on the other hand that they are related to second-gradient material coefficients. In this section we show a result, on the pantographic structure of Fig. 17, that makes explicit the experimental evidence of such a boundary layer. Besides, by using the second gradient model of dell'Isola et al. (2016), we show that it is possible to characterize the largeness of the boundary layer in terms of the second gradient coefficient (i.e., $K_{I I}$ ) of that model, that means that a simple first gradient model (i.e., a model with $\left.K_{I I}=0\right)$ is not sufficient to predict the correct experimental evidence. 


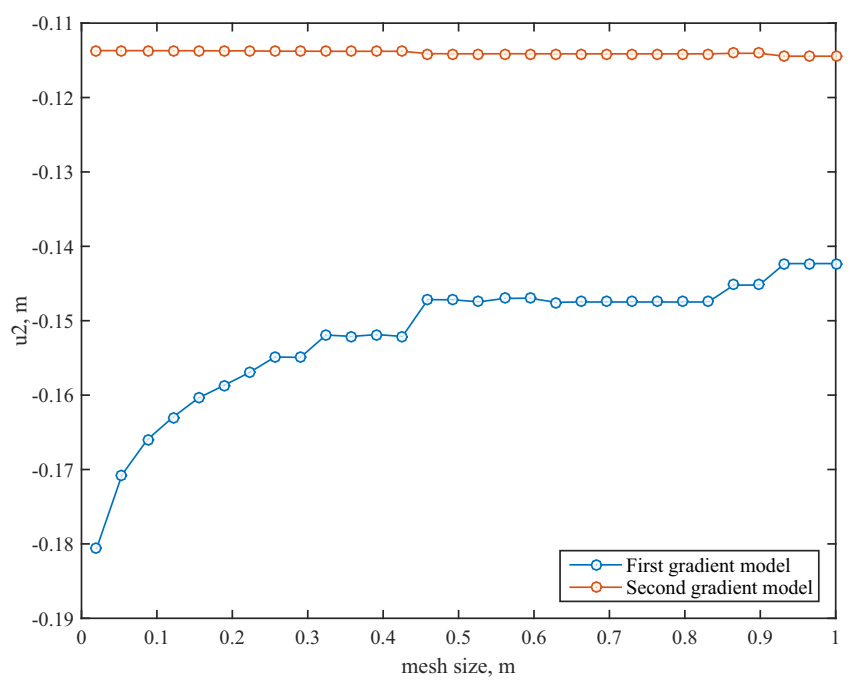

Fig. 16. Comparison between the vertical displacement at the center of side $S$ in the (a) first and (b) second gradient models in the case of an external vertical wedge force at $V_{3}$.

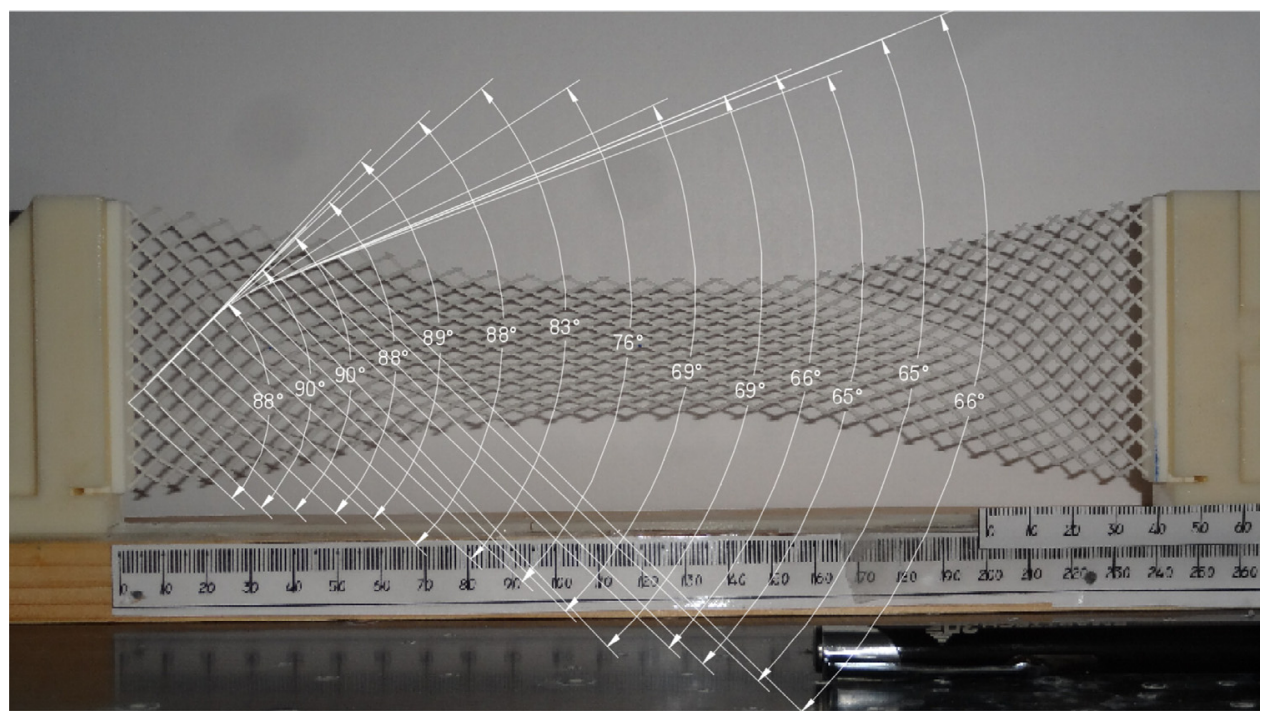

Fig. 17. A bias test on a standard pantographic structure is shown. The angles across the two families of fibers in the deformed configuration are evaluated by image analysis.

The elastic non-linear anisotropic internal energy density of the model that is used in dell'Isola et al. (2016) to numerically evaluate the shear angles that are shown in Fig. 18 is the following,

$$
\begin{aligned}
U(F, \nabla F)= & \sum_{\alpha=1}^{2}\left\{\frac{K_{e}}{2}\left(F_{a b} D_{b}^{\alpha}-1\right)\left(F_{a c} D_{c}^{\alpha}-1\right)\right. \\
& \left.+\frac{K_{I I}}{2}\left[\frac{F_{a b, c} F_{a d, e} D_{b}^{\alpha} D_{c}^{\alpha} D_{d}^{\alpha} D_{e}^{\alpha}}{F_{f g} F_{f h} D_{g}^{\alpha} D_{h}^{\alpha}}-\left(\frac{F_{a b} F_{a c, d} D_{b}^{\alpha} D_{c}^{\alpha} D_{d}^{\alpha}}{F_{f g} F_{f h} D_{g}^{\alpha} D_{h}^{\alpha}}\right)^{2}\right]\right\} \\
& +\frac{K_{b}}{2}\left[\arccos \frac{F_{a b} F_{a c} D_{b}^{1} D_{c}^{2}}{\sqrt{F_{d e} F_{d f} D_{e}^{1} D_{f}^{1}} \sqrt{F_{g h} F_{g i} D_{h}^{2} D_{i}^{2}}}-\frac{\pi}{2}\right]^{\gamma}
\end{aligned}
$$

where the two families of fibers are initially directed along the two orthogonal unit vectors $\mathbf{D}^{1}$ and $\mathbf{D}^{2}$ and where the material coefficients that have been used are $K_{e}=0.134 \mathrm{MN} / \mathrm{m}, K_{p}=159 \mathrm{~N} / \mathrm{m}$ and $\gamma=1.36$. We also remark that the bias 


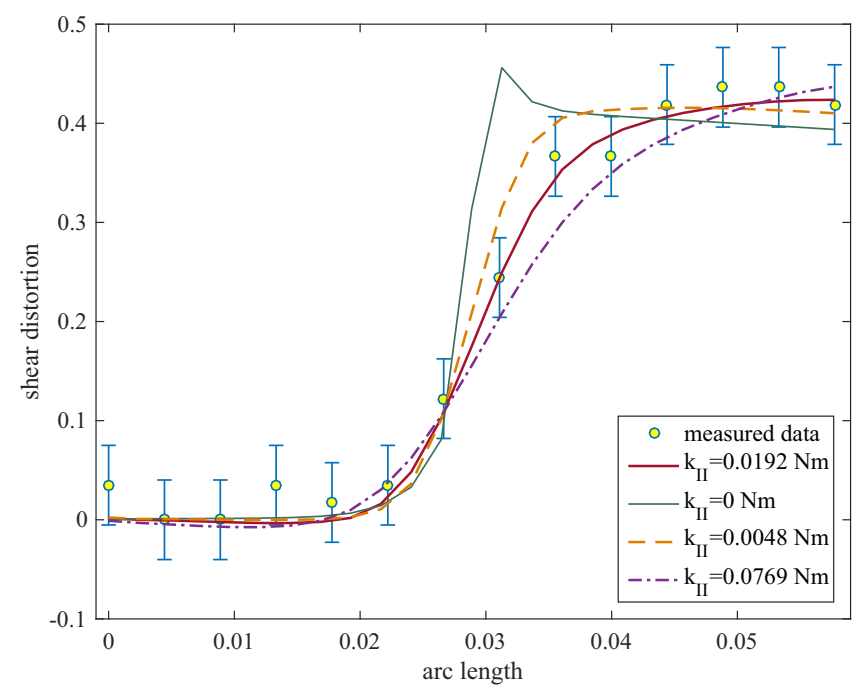

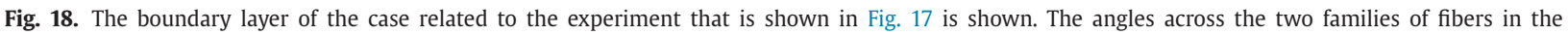

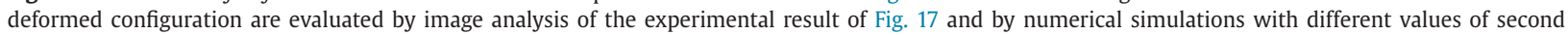
gradient coefficients $K_{I I}$.

test that is shown in Fig. 17 is accomplished by imposing a displacement, towards the direction parallel to the long side of the rectangle, of the short-side of the rectangle, that in turn is directed at $\pi / 4$ with respect to the two orthogonal unit vectors $\mathbf{D}^{1}$ and $\mathbf{D}^{2}$. In the deformed configuration the angle $\varphi$ between the two families of fibers is not anymore at $\pi / 2$. It is

$$
\varphi=\arccos \frac{F_{a b} F_{a c} D_{b}^{1} D_{c}^{2}}{\sqrt{F_{d e} F_{d f} D_{e}^{1} D_{f}^{1}} \sqrt{F_{g h} F_{g i} D_{h}^{2} D_{i}^{2}}},
$$

and the shear angle $\phi$ (or shear deformation in Fig. 18) is simply

$$
\phi=\varphi-\frac{\pi}{2} \text {. }
$$

If we evaluate the shear angle $\phi$ along the arc-length that is shown in Fig. 17, it is almost zero near short side of the rectangle and reach a finite value by passing through a boundary layer. The largeness of such a boundary layer is related to the second gradient parameter $K_{I I}$.

In Fig. 18 we show that the optimal value for the second gradient constitutive parameter $K_{I I}$ is $K_{I I}=0.0192 \mathrm{Nm}$. However, we also shown that different values of this parameter give a wrong largeness of the boundary layer. In particular we observe that a reduction $1 / 4$ of the second gradient constitutive parameter $K_{I I}$ give a smaller boundary layer and a magnification of 4 give a larger boundary layer. Finally, a numerical simulation in which the second gradient contribution of the strain energy (73) is assumed to vanish, i.e. $K_{I I}=0$, produce no-boundary layer. Besides, numerically instability is observed in this last case.

\section{Conclusions}

A perfect overlap of numerical simulations obtained with a commercial code and closed form solution of selected classical benchmark boundary value problems have been found and reported in this paper. The role of external double and wedge forces has also been presented. Besides, we show a mesh-independent behaviour of second gradient numerical solution with respect to the corresponding first gradient counterpart. Finally, we show an experimental bias test on a specific pantographic structure and extrapolate an internal boundary layer in terms of the shear angles across initially orthogonal fibers. A non-linear anisotropic model is also presented aimed to reproduce the shown experimental results. In particular, we exhibit comparisons between the numerical simulation of the proposed theoretical model and the experimental results in terms of the internal boundary layer. Such a comparison has permitted to identify the second gradient coefficient of this model.

\section{References}

Akarapu, S., \& Zbib, H. M. (2006). Numerical analysis of plane cracks in strain-gradient elastic materials. International Journal of Fracture, 141(3-4), 403-430. doi:10.1007/s10704-006-9004-y. 
Alibert, J.-J., \& Corte, A. D. (2015). Second-gradient continua as homogenized limit of pantographic microstructured plates: A rigorous proof. Zeitschrift für angewandte Mathematik und Physik, 66.5, 2855-2870.

Altenbach, H., Eremeyev, V. A., \& Morozov, N. F. (2010). On equations of the linear theory of shells with surface stresses taken into account. Mechanics of Solids, 45(3), 331-342.

Aminpour, H., \& Rizzi, N. (2015). On the continuum modelling of carbon nano tubes. In Civil-Comp Proceedings Volume 108.

Aminpour, H., \& Rizzi, N. (2016a). On the modelling of carbon nano tubes as generalized continua. Advanced Structured Materials, 42, 15-35.

Aminpour, H., \& Rizzi, N. (2016b). A one-dimensional continuum with microstructure for single-wall carbon nanotubes bifurcation analysis. Mathematics and Mechanics of Solids, 21(2), 168-181.

Aminpour, H., Rizzi, N., \& Salerno, G. (2014). A one-dimensional beam model for single-wall carbon nano tube column buckling. In Civil-Comp Proceedings. Volume 106.

Andreaus, U., Giorgio, I., \& Lekszycki, T. (1 December 2014). A 2-d continuum model of a mixture of bone tissue and bio-resorbable material for simulating mass density redistribution under load slowly variable in time. ZAMM Zeitschrift fur Angewandte Mathematik und Mechanik, 94(12), 978-1000.

Argyris, J. H., Fried, I., \& Scharpf, D. W. (1968). The TUBA family of plate elements for the matrix displacement method. Aeronautical Journal of the Royal Aeronautical Society, 72(692), 701-709.

Assante, D., \& Cesarano, C. (2014). Simple semi-analytical expression of the lightning base current in the frequency-domain. Journal of Engineering Science and Technology Review, 7(2), 1-6.

Atluri, S. N., \& Cazzani, A. (March 1995). Rotations in computational solid mechanics. Archives of Computational Methods in Engineering, 2(1), 49-138.

Auffray, N., Bouchet, R., \& Brechet, Y. (2009). Derivation of anisotropic matrix for bi-dimensional strain-gradient elasticity behavior. International Journal of Solids and Structures, 46.2, 440-454.

Auffray, N., dell'Isola, F., Eremeyev, V. A., Madeo, A., \& Rosi, G. (4 April 2015). Analytical continuum mechanics à la hamilton-piola least action principle for second gradient continua and capillary fluids. Mathematics and Mechanics of Solids, 20(4), 375-417.

Bilotta, A., Formica, G., \& Turco, E. (September 2010). Performance of a high-continuity finite element in three-dimensional elasticity. International Journal for Numerical Methods in Biomedical Engineering, 26(9), 1155-1175.

Bilotta, A., \& Turco, E. (2009). A numerical study on the solution of the cauchy problem in elasticity. International Journal of Solids and Structures, 46(25-26), 4451-4477.

Bulíček, M., Málek, J., Rajagopal, K. R., \& Walton, J. R. (2015). Existence of solutions for the anti-plane stress for a new class of "strain-limiting" elastic bodies. Calculus of Variations and Partial Differential Equations, 54(2), 2115-2147.

Carcaterra, A., \& Roveri, N. (2012). Energy distribution in impulsively excited structures. Shock and Vibration, 19(5), 1143-1163.

Cesarano, C. (2014). Generalized chebyshev polynomials. Hacettepe Journal of Mathematics and Statistics, 43(5), 731-740.

Cesarano, C., \& Assante, D. (2014). A note on generalized bessel functions. International Journal of Mathematical Models and Methods in Applied Sciences, 8(1), $38-42$.

Cesarano, C., Fornaro, C., \& Vazquez, L. (2016). Operational results on bi-orthogonal hermite functions. Acta Mathematica Universitatis Comenianae, 85(1), 43-68.

Cuomo, M., Contrafatto, L., \& Greco, L. (July 2014). A variational model based on isogeometric interpolation for the analysis of cracked bodies. International Journal of Engineering Science, 80, 173-188.

Dasgupta, S., \& Sengupta, D. (1990). A higher-order triangular plate bending element revisited. International Journal for Numerical Methods in Engineering, 30, 419-430. doi:10.1002/nme.1620300303.

Del Vescovo, D., \& Giorgio, I. (2014). Dynamic problems for metamaterials: Review of existing models and ideas for further research. International Journal of Engineering Science, 80, 153-172.

dell'Isola, F., Giorgio, I., Pawlikowski, M., \& Rizzi, N. L. (2016). Large deformations of planar extensible beams and pantographic lattices: Heuristic homogenization, experimental and numerical examples of equilibrium. Proceedings of the Royal Society A: Mathematical, Physical and Engineering Sciences, 472(2185). Article number 20150790 pages (23)

dell'Isola, F., Guarascio, M., \& Hutter, K. (2000). A variational approach for the deformation of a saturated porous solid. a second-gradient theory extending Terzaghi's effective stress principle. Archive of Applied Mechanics, 70(5), 323-337.

dell'Isola, F., Madeo, A., \& Placidi, L. (2012). Linear plane wave propagation and normal transmission and reflection at discontinuity surfaces in second gradient 3d continua. ZAMM -Journal of Applied Mathematics and Mechanics / Zeitschrift für Angewandte Mathematik und Mechanik, 92(1), 52-71.

Federico, S., Grillo, A., \& Herzog, W. (2004). A transversely isotropic composite with a statistical distribution of spheroidal inclusions: A geometrical approach to overall properties. Journal of the Mechanics and Physics of Solids, 52, 2309-2327.

Federico, S., Grillo, A., \& Imatani, S. (2015). The linear elasticity tensor of incompressible materials. Mathematics and Mechanics of Solids, 20(6), 643-662.

Federico, S., Grillo, A., \& Wittum, G. (2009). Considerations on incompressibility in linear elasticity. Nuovo Cimento C, 32C, 81-87.

Ferretti, M., Madeo, A., dell'Isola, F., \& Boisse, P. (2014). Modelling the onset of shear boundary layers in fibrous composite reinforcements by second gradient theory. ZAMP-Zeitschrift für angewandte Mathematik und Physik, 65(3), 587-612.

Fischer, P., Klassen, M., Mergheim, J., Steinmann, P., \& Müller, R. (2011). Isogeometric analysis of 2d gradient elasticity. Computational Mechanics, 47, $325-334$.

Fischer, P., Mergheim, J., \& Steinmann, P. (2010). On the c1 continuous discretization of non-linear gradient elasticity: A comparison of NEM and FEM based on bernstein-bézier patches. International Journal for Numerical Methods in Engineering, 82(10), 1282-1307. doi:10.1002/nme.2802.

Gabriele, S., Rizzi, N., \& Varano, V. (2012). On the imperfection sensitivity of thin-walled frames. Civil-comp proceedings .volume 99, 2012 11th international conference on computational structures technology, CST. Dubrovnik; Croatia; 4 September 2012 through 7 September 2012; Code 102644

Gabriele, S., Rizzi, N., \& Varano, V. (2016). A 1d nonlinear TWB model accounting for in plane cross-section deformation. International Journal of Solids and Structures, 94-95, 170-178.

Gabriele, S., Rizzi, N. L., \& Varano, V. (2014). A one-dimensional nonlinear thin walled beam model derived from koiter shell theory. Civil-comp proceedings volume 106.

Garusi, E., Tralli, A., \& Cazzani, A. (2004). An unsymmetric stress formulation for reissner-mindlin plates: A simple and locking-free rectangular element. International Journal of Computational Engineering Science, 5(3), 589-618.

Greco, L., \& Cuomo, M. (2015). Consistent tangent operator for an exact kirchhoff rod model. Continuum Mechanics and Thermodynamics, 27(4-5), 861-877.

Hill, R. (1965). A self-consistent mechanics of composite materials. Journal of the Mechanics and Physics of Solids, 13, 213-222.

Ionel-Dumitrel, G., Patrizio, N., Angela, M., Placidi, L., \& Giuseppe, R. (2015). The relaxed linear micromorphic continuum: Existance, uniqueness and continuous dependence in dynamics. Mathematics and Mechanics of Solids, 20, 1171-1197. doi:10.1177/1081286513516972. ISSN: 1081-2865.

Javili, A., Dell'Isola, F., \& Steinmann, P. (2013). Geometrically nonlinear higher-gradient elasticity with energetic boundaries. Journal of the Mechanics and Physics of Solids, 61(12), 2381-2401.

Luca, P. (2015). A variational approach for a nonlinear 1-dimensional second gradient continuum damage model. Continuum Mechanics and Thermodynamics, 27, 623-638. doi:10.1007/s00161-14-0338-9. ISSN:0935-1175

Luca, P. (2016). A variational approach for a nonlinear one-dimensional damage-elasto-plastic second-gradient continuum model. Continuum Mechanics and Thermodynamics, 28, 119-137. doi:10.1007/s00161-014-0405-2. ISSN: 0935-1175

Luca, P., Andreaus, U., Corte, A. D., \& Lekszycki, T. (2015). Gedanken experiments for the determination of two-dimensional linear second gradient elasticity coefficients. Zeitschrift fur Angewandte Mathematik und Physik, 66, 3699-3725. doi:10.1007/s00033-015-0588-9. ISSN: 0044-2275.

Madeo, A., dell'Isola, F., \& Darve, F. (2013). A continuum model for deformable, second gradient porous media partially saturated with compressible fluids. Journal of the Mechanics and Physics of Solids, 61(11), 2196-2211.

Madeo, A., Neff, P., Ghiba, I.-D., Placidi, L., \& Rosi, G. (2015a). Band gaps in the relaxed linear micromorphic continuum. Zeitschrift Für Angewandte Mathematik und Mechanik, 95, 880-887. doi:10.1002/zamm.201400036. ISSN:1521-4001. 


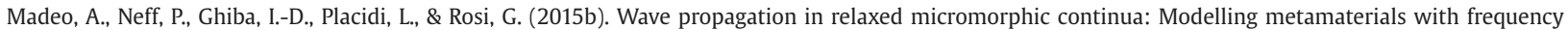
band-gaps. Continuum Mechanics and Thermodynamics, 27, 551-570. doi:10.1007/s00161-013-0329-2. ISSN: 0935-1175.

Mareno, A., \& Healey, T. J. (2006). Global continuation in second-gradient nonlinear elasticity. SIAM Journal on Mathematical Analysis, 38(1), 103-115.

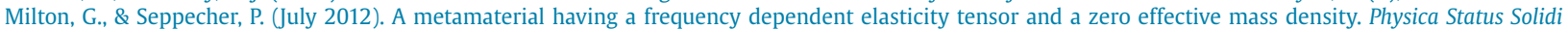
(B) Basic Research, 249(7), 1412-1414.

Mindlin, R. D. (1936). Force at a point in the interior of a semi-infinite solid. Journal of Applied Physics, 7(5), 195-202.

Mindlin, R. D. (1964). Micro-structure in linear elasticity. Department of Civil Engineering Columbia University New York 27, New York.

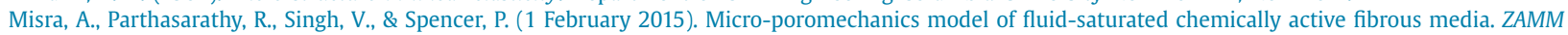
Zeitschrift fur Angewandte Mathematik und Mechanik, 95(2), 215-234.

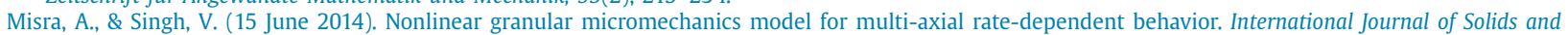
Structures, 51(13), 2272-2282.

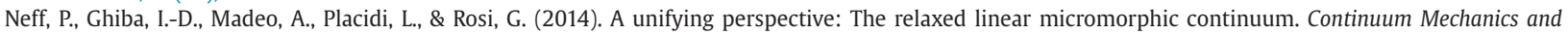
Thermodynamics, 26, 639-681. doi:10.1007/s00161-013-0322-9. ISSN: 0935-1175.

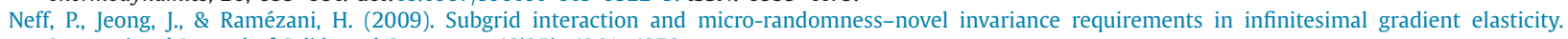
International Journal of Solids and Structures, 46(25), 4261-4276.

de Oliveira Góes, R. C., de Castro, J. T. P., \& Martha, L. F. (2014). 3D effects around notch and crack tips. International Journal of Fatigue, 62, 159-170.

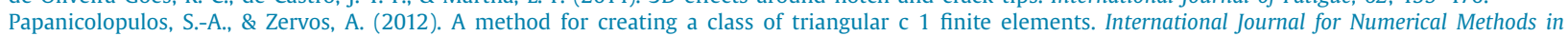
Engineering, 89(11), 1437-1450.

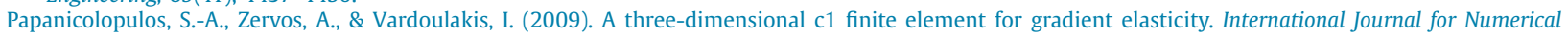
Methods in Engineering, 77(10), 1396-1415.

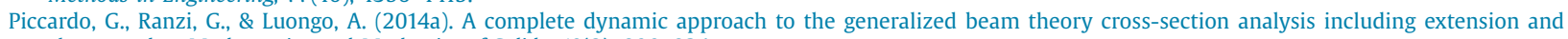
shear modes. Mathematics and Mechanics of Solids, 19(8), 900-924.

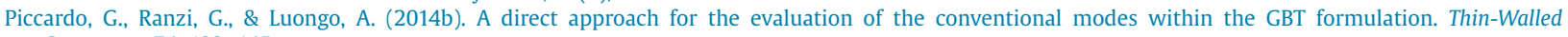
Structures, 74, 133-145.

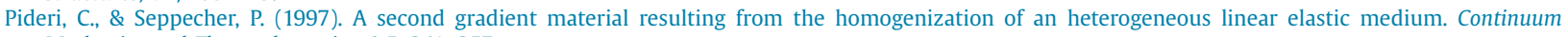
Mechanics and Thermodynamics, 9.5, 241-257.

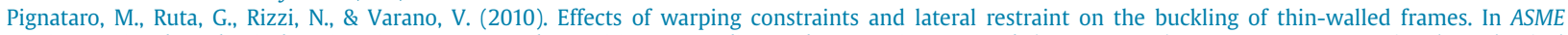
international mechanical engineering congress and exposition, proceedings volume 10, issue PART b (pp. 803-810). ASME 2009 International Mechanical Engineering Congress and Exposition, IMECE2009; Lake Buena Vista, FL; United States; 13 November 2009 through 19 November 2009 ; Code 80879

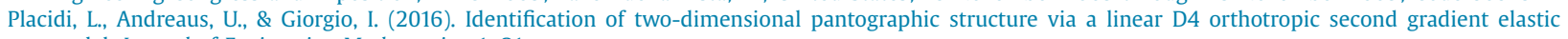
model. Journal of Engineering Mathematics, 1-21.

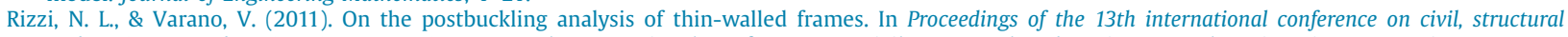
and environmental engineering computing. 14p 13th International Conference on Civil, Structural and Environmental Engineering Computing, CC 2011; Chania, Crete; Greece; 6 September 2011 through 9 September 2011; Code 89029.

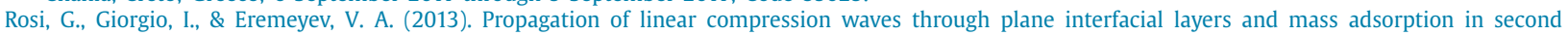
gradient fluids. ZAMM Zeitschrift fur Angewandte Mathematik und Mechanik, 93(12), 914-927.

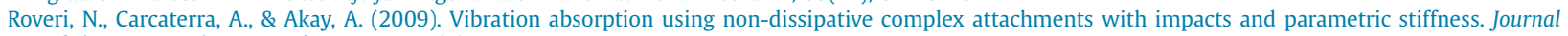
of the Acoustical Society of America, 126(5), 2306-2314.

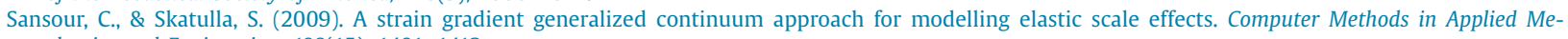
chanics and Engineering, 198(15), 1401-1412.

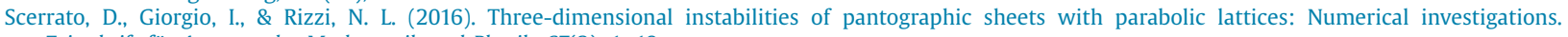
Zeitschrift für Angewandte Mathematik und Physik, 67(3), 1-19.

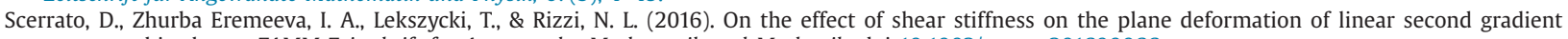
pantographic sheets. ZAMM Zeitschrift fur Angewandte Mathematik und Mechanik. doi:10.1002/zamm.201600066.

Sciarra, G., dell'Isola, F., \& Coussy, O. (2007). Second gradient poromechanics. International Journal of Solids and Structures, 44(20), 6607-6629.

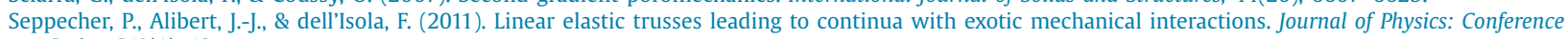
Series, 319(1), 13pages.

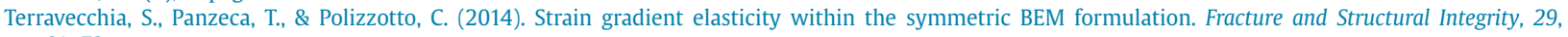
61-73.

Walpole, L. J. (1981). Elastic behavior of composite materials: Theoretical foundations. Advances in Mechanics, 21, 169-242.

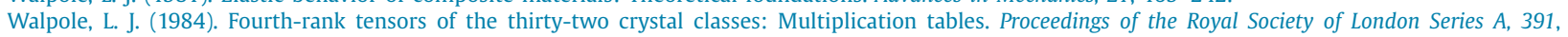
149-179.

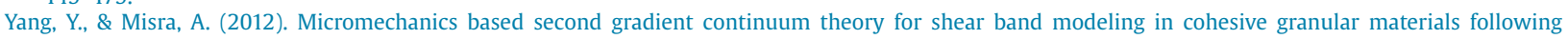
damage elasticity. International Journal of Solids and Structures, 49(18), 2500-2514.

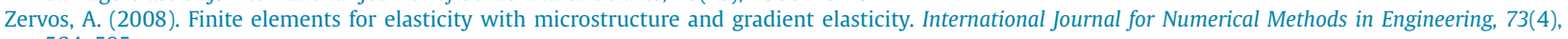
564-595.

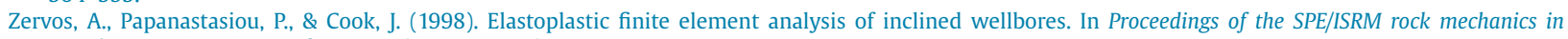
petroleum engineering conference, 1 (pp. 535-544).

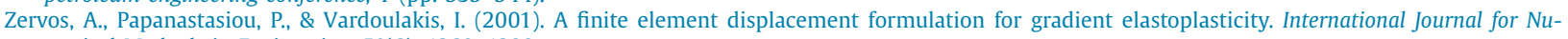
merical Methods in Engineering, 50(6), 1369-1388.

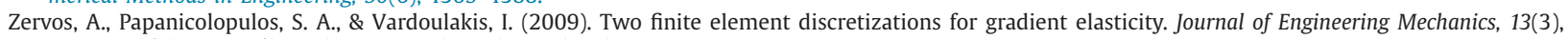
203-213. doi:10.1061/(ASCE)0733-9399(2009)135:3(203).

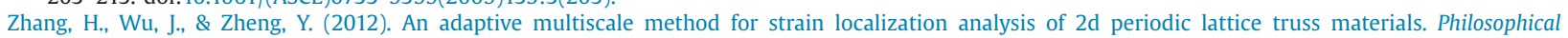
Magazine, 92(28-30), 3723-3752. 